\title{
Generation, characterisation, and applications of atomic and molecular alignment and orientation
}

\author{
Claire Vallance \\ Department of Chemistry, University of Oxford, \\ Chemistry Research Laboratory, 12 Mansfield Rd, Oxford OX1 3TA, UK \\ *corresponding author: claire.vallance@chem.ox.ac.uk
}

\begin{abstract}
The gas phase is generally defined as a state of matter in which atoms or molecules are in constant, rapid, random Brownian motion. However, a range of techniques exist for preparing distributions of gas phase atoms and molecules whose motion is far from random, and whose orientation in space is well defined. In this Perspective, we will explore the nature of atomic and molecular alignment and orientation, the various techniques by which samples of spatially oriented species may be prepared and characterised, and some of the ways in which oriented molecules are being exploited to further our knowledge of molecular structure and dynamics.
\end{abstract}




\section{Introduction}

Any general definition of a gas will describe a substance that expands to fill any container, and which at a microscopic level is made up of atoms or molecules with a broad distribution of velocities, each undergoing completely random motion as a consequence of constant collisions with its neighbours. For physical chemists, the gas phase is a favourite playground as it offers the opportunity to study molecules which are essentially isolated in space, and whose behaviour is therefore not perturbed by intermolecular interactions as would be the case in liquid or solid phases. Unfortunately, for a gas in its 'native' state this comes at the cost of averaging over all molecular orientations and over a broad velocity distribution. Not content with the limited amount of information such experiments can provide, much effort has been directed towards controlling the behaviour of molecules in the gas phase. Early on, the advent of molecular beams tamed the velocity distribution, allowing gas-phase molecules to be prepared in a collision free environment with a well-defined velocity. More recently, a variety of techniques have been developed which provide partial or complete control over molecular orientation. In addition to paving the way for a wide range of experiments in which stereochemical effects on chemical reactivity are probed at the single-collision level, such developments hint at the tantalising prospect of future electron or X-ray diffraction studies on single molecules in the gas phase. In the following pages, we will review the wide range of approaches to controlling the motion and orientation of gas phase molecules, before exploring some of the applications of oriented chemical species.

\subsection{Controlling the translational motion of a gas: molecular beams}

With a few exceptions, the first stage in creating a distribution of oriented atoms or molecules is to synchronise their translational motion. This is readily achieved by preparing the sample in a molecular beam via a supersonic jet expansion. A molecular beam is formed when gas from a containment reservoir flows through an orifice into a vacuum. The flow characteristics of the beam depend on the ratio of the mean free path of the gas inside the source, $\lambda$, to the orifice diameter, $\phi$, a quantity known as the Knudsen number.

$$
K_{n}=\frac{\lambda}{\phi}
$$


A supersonic expansion requires $K_{n}<1$, and yields a high intensity molecular beam with a narrow velocity distribution. Because the nozzle diameter is greater than the mean free path of the molecules inside the source, multiple collisions occur as the gas passes through the throat of the nozzle. Successful collisions transfer momentum to the axial direction, giving a high velocity along the axis and a small random velocity perpendicular to the axis. The collisions also convert the internal energy and randomly directed translational energy of the molecules inside the source into kinetic energy of translational motion along the beam axis, with the consequence that the beam molecules are internally cold. A simple thermodynamic model, in which it is assumed that the total enthalpy of the gas inside the source is converted into translational kinetic energy of the beam, may be used to calculate the maximum or terminal velocity $u_{\max }$ of the beam molecules [1].

$$
u_{\max }=\left(\frac{2 k_{\mathrm{B}} T_{0}}{m} \frac{\gamma}{\gamma-1}\right)^{1 / 2}
$$

where $T_{0}$ is the temperature inside the source, $m$ is the atomic or molecular mass, and $\gamma=C_{p} / C_{V}$ is the ratio of the heat capacities at constant pressure and volume for the beam gas. The beam velocity may be controlled by 'seeding' the species of interest into an inert carrier gas. The final velocity of the beam is then determined by the average mass and heat capacity of the gases in the mixture. Seeding a molecule of molecular mass $m$ into a carrier gas with a molecular mass greater than $m$ results in a lower flow velocity than that for a pure beam, while seeding into a lighter gas gives a higher velocity. The ratio of seed to carrier gas can be altered to give beam velocities which span the range between the terminal velocities of the seed and carrier gas.

\subsection{Defining atomic and molecular orientation and alignment}

The preceding section explains how to prepare a sample of gas-phase atoms or molecules moving in a well-defined direction with a well-defined velocity. In Section 2 we will explore ways in which the atoms or molecules within a molecular beam may be oriented in space, but first we need to consider in more detail what we mean by orientation in this context.

Most generally, a distribution of atoms or molecules which are not randomly oriented in space is said to be polarised. Intuitively, we might think of an oriented molecule as a stationary entity in which all of the constituent atoms are located at well-defined positions in space, as shown in Figure 1(a). As we shall see later, state-of-the-art laser-based techniques allow us to come very close to 
achieving this level of orientation. However, many other methods for orienting molecules are not able to freeze out rotational motion completely, and we therefore need to consider how to define the polarisation of a distribution of rotating molecules. Often, the most straightforward way to do this is simply to consider the polarisation of the angular momentum vector of the molecules, which defines their axis of rotation. Figure 1 (b) shows a hypothetical distribution of rotating molecules which are all identically oriented in space.

(a)

(b)
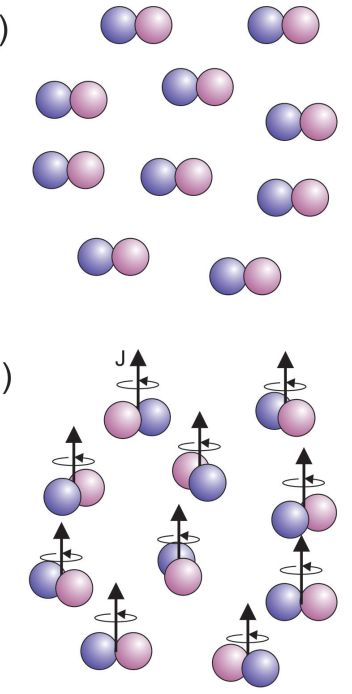

(c)
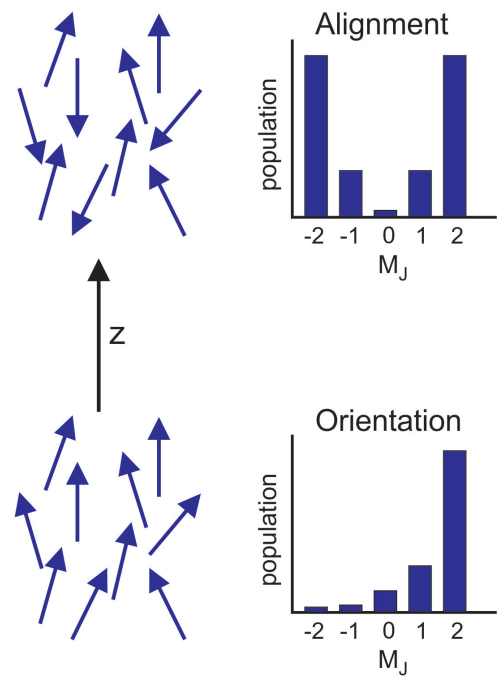

Figure 1: (a) orientation of bond axes; (b) orientation of rotational angular momentum; (c) classical and quantum mechanical descriptions of alignment and orientation.

While the total angular momentum of a closed-shell molecule arises purely from its rotational motion, in an open-shell molecule there may also be a contribution from electronic angular momentum. Defining spatial polarisation in terms of the total angular momentum vector also provides a straightforward way to treat polarisation in atoms, in which the only contribution to the total angular momentum is electronic, in the form of spin and electronic orbital angular momentum.

Polarisation of a distribution of vectors, in this case atomic or molecular angular momentum vectors, is often subdivided into two categories: alignment and orientation [2]. In this context, 'alignment' and 'orientation' have very precise definitions. An aligned distribution of vectors is one in which all of the vectors are aligned along a single axis (or sometimes in a plane) but with no preferred direction i.e. in the aligned distribution in Figure 1(c), the arrows are preferentially aligned along the vertical axis, but there are equal numbers of arrows pointing in the up and down directions. An oriented distribution, on the other hand, is one in which the vectors have 
both a preferred axis and a preferred direction; in the oriented distribution in Figure 1(c), the arrows again have a preferred axis, but now they are all pointing upwards. When dealing with a quantum mechanical angular momentum vector $\mathbf{J}$, whose direction is defined by the quantum number $M_{J}$, alignment implies an anisotropic distribution of $\left|M_{J}\right|$, while orientation implies an anisotropic distribution of $M_{J}$. Example $M_{J}$ distributions for the two cases are shown to the right of Figure 1(c).

While it is often most straightforward to consider alignment or orientation of angular momentum, we should note that if the angular momentum vector of a molecule is oriented in space, the spatial distribution of one or more bonds will generally also show significant polarisation. For example, if the rotational angular momentum vector of a diatomic molecule is aligned along the $z$ axis, the bond axis will lie preferentially in the $x y$ plane, and not along $z$. Depending on the context, in the following we shall refer to polarisation in terms of both angular momentum polarisation and bond axis polarisation.

\subsection{Quantifying atomic and molecular polarisation}

Bringing together the various aspects of control we have considered thus far, to specify the polarisation of an individual atom or molecule completely, we must specify both its velocity vector $\mathbf{v}$ and its total angular momentum vector $\mathbf{J}$. The polarisation of an ensemble of atoms or molecules is therefore described by a probability distribution involving these two vectors, $P(\mathbf{v}, \mathbf{J})$, or, equivalently, $P\left(\theta_{v}, \phi_{v} ; \theta_{J}, \phi_{J}\right)$, where $\theta_{v}, \phi_{v}, \theta_{J}$, and $\phi_{J}$ are the polar angles describing the directions of $\mathbf{v}$ and $\mathbf{J}$, respectively. In some cases, such as the strong laser field alignment studies described in Section 2.3, it is sufficient simply to describe the orientation and alignment in terms of the expectation values $\langle\cos \theta\rangle$ or $\left\langle\cos ^{2}(\theta)\right\rangle$, of the molecular axis about the field direction. In the field of photodissociation stereodynamics, quantifying atomic or molecular alignment and orientation generally consists of expanding the double vector distribution described above in a basis set of suitable functions. Quantifying the polarisation then reduces to determining the expansion coefficients i.e. the expansion coefficients comprise a set of polarisation parameters that completely define the polarisation distribution of the ensemble. The polarisation parameters may also be used to calculate the $M_{J}$ populations that characterise the quantised orientation of $\mathbf{J}$. Rather unfortunately, there is no single set of agreed polarisation parameters; instead, a number of different formalisms have 
been developed in parallel, with each having a faithful band of supporters and detractors. Fortunately, it is often relatively straightforward to transform from one set of polarisation parameters to another in order to compare experimental results [10]. Descriptions in widespread use include Dixon's semiclassical bipolar moment expansion [3]; Rakitzis and Zare's approach [4], in which spherical harmonic expansions are used to describe contributions to the polarisation arising from parallel, perpendicular, and mixed components of an optical transition; and the state multipole formalism adopted by Vasyutinksi and Suits[5]. The latter two of these formalisms have their origins in the full quantum description provided in the mid 1990s by Siebbeles et al[6]. More recently, Aoiz and Miranda have shed light on the relationship between classical and quantum mechanical descriptions of angular momentum polarisation, presenting a unified treatment of the two approaches $[7,8]$. Finally, when studying orientation and alignment arising as a result of chemical reaction, the most popular approach employs the polarisation-dependent differential cross section methodology developed by Shafer-Ray et al. [9].

Many different data fitting and analysis schemes have been developed in order to extract polarisation parameters from experimental data, and we will make no attempt to summarise these here, focusing instead on the various general approaches available for generating, characterising, and exploiting spatially polarised molecules.

\section{Generation of polarised atoms and molecules}

For some molecules, we obtain a considerable degree of spatial alignment 'for free' during the supersonic expansion used to prepare the molecules in a molecular beam. This phenomenon is described in detail by Hershbach and coworkers [11], and by Aquilanti and coworkers [12, 13, 14], amongst others $[15,16]$. At a basic level the origin of the alignment is straightforward to understand, arising as a consequence of the large number of collisions a molecule undergoes during the supersonic expansion. Molecules in orientations and rotational states that present a large collision cross section when viewed along the beam direction undergo a larger number of collisions than those presenting a smaller cross section. Since collisions alter the molecular orientation, over the many collisions that occur during the expansion the high-cross-section orientations are gradually converted into lowcross-section orientations. As a result, linear molecules tend to fly 'end on' rather than 'side on', 
while planar molecules, such as benzene, tend to fly 'side on' rather than 'face on'. The phenomenon is strongly velocity-dependent across the beam velocity distribution. The slowest molecules, which have undergone fewest collisions during the expansion, show virtually no alignment, while the fastest components of the beam, which have undergone the most collisions, show marked alignment. As an example, the beam velocity distribution and degree of alignment for $\mathrm{O}_{2}$ seeded in Helium is shown in Figure 2.

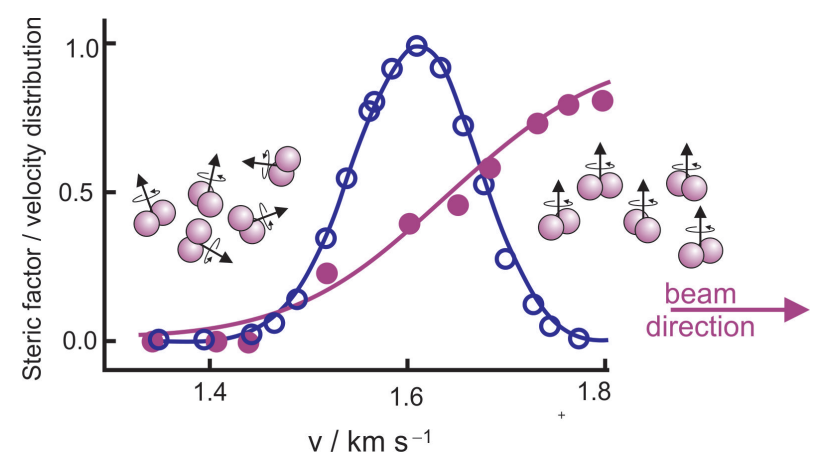

Figure 2: Velocity distribution (open circles) and steric factor (closed circles) for $\mathrm{O}_{2}$ seeded $2.5 \%$ in He. The steric factor, $P$, is defined as $P=\left(w_{\perp}-w_{\|}\right) /\left(w_{\perp}+w_{\|}\right)$, where $w_{\perp}$ and $w_{\|}$are the weights for $\mathrm{O}_{2}$ molecules with their rotational angular momentum aligned perpendicular to and parallel to the beam axis, respectively. Adapted from reference [12]

In order to generate a more strongly polarised distribution of atoms or molecules from a randomly oriented distribution, some vector property of the atom or molecule must be used as a 'handle' that can interact with an external force in order to induce alignment or orientation. If a molecule has a suitably strong permanent dipole moment, we can exploit its interaction with an external electric field to induce spatial polarisation. More generally, we can induce alignment or orientation by exploiting the interaction of transition dipoles within the atom or molecule with linearly or circularly polarised laser light. We explore these approaches, some of which have been reviewed previously by Orr-Ewing [16], in more detail in the following.

\section{1 'Brute force' alignment}

The simplest means of orienting a dipolar molecule is to place it in a uniform electric field $\mathbf{E}$ of sufficient strength that the interaction energy $-\mu \cdot \mathbf{E}$ between the dipole $\mu$ and the electric field 
greatly exceeds the rotational energy of the molecule i.e. $\frac{\mu E}{B}>>1$, where $B$ is the rotational constant of the molecule. When this condition is satisfied, the rotational states of the molecules are transformed into so-called 'pendular' or librational states, essentially frustrated rotations in which the dipole moment oscillates like a pendulum about the field direction. While 'brute force' orientation, as this technique was christened, is in principle almost entirely general and applicable to any polar or polarisable molecule, achieving significant orientation in this way requires high electric field strengths, on the order of $\sim 100 \mathrm{kV} \mathrm{cm}^{-1}$, and a molecule with a large dipole moment and small rotational constant. In addition, since the rotational energy increases quadratically with rotational quantum number $J$, the molecules must be rotationally extremely cold, which generally means they must be prepared in a supersonic expansion. Brute force orientation was first demonstrated in 1990 by Loesch and Remscheid [17], and has since been applied to a range of molecules, including $\mathrm{CH}_{3} \mathrm{I}$, ICl, ICN, and pyradazine $[17,18,19,20]$.

\subsection{Quadrupole and hexapole state selection and orientation}

In contrast to the extremely strong homogeneous electric fields used in the brute force orientation method, quadrupole and hexapole state selectors exploit the trajectories of dipolar molecules through a weaker inhomogeneous electric field to achieve spatial orientation of diatomic and symmetric top molecules, respectively. When a molecule with a permanent dipole is subjected to an external field, its energy is lowered by the Stark effect if its dipole is aligned parallel to the field (a lower Stark state), and raised if its dipole is aligned antiparallel to the field (an upper Stark state). In an inhomogeneous electric field, lower Stark states can lower their energy by moving to regions of higher field - and for this reason are sometimes known as 'high-field-seeking states' while upper Stark states can lower their energy by moving to regions of lower field, and are also known as 'low-field-seeking states'.

Quadrupole and hexapole state selectors consist of four or six rods mounted on an inscribed radius $r_{0}$, with positive and negative high voltages applied to alternate rods. While the general principles of state selection and orientation are very similar for diatomics in a quadrupole and symmetric tops in a hexapole, they differ in the details. Quadrupole state selection and orientation is limited to a small number of diatomic molecules with very large dipole moments, and is discussed in detail by Toennies and coworkers [21]. Here we will focus on the hexapole state selector. This 
(a)

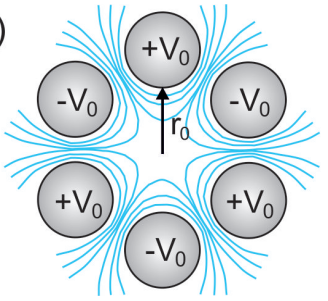

(b)

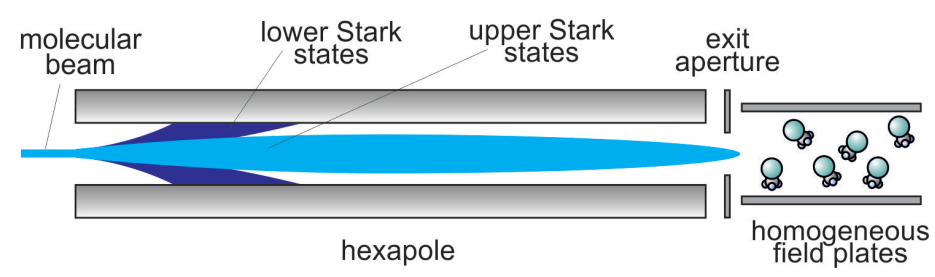

Figure 3: (a) Inhomogeneous electric field generated by a hexapole. The inscribed radius $\mathrm{r}_{0}$ is shown. (b) Principle of hexapole state selection and orientation: the hexapole selects upper Stark states from the initially randomly oriented molecular beam. These then pass adiabatically into a region of uniform electric field in order to achieve orientation in the lab frame.

has been used to prepare spatially oriented samples of a wide range of molecules, including the methyl halides, $\mathrm{NO}, \mathrm{OH}$, and even, recently, the asymmetric top propylene oxide [22].

The rotational state of a symmetric top molecule is defined by three quantum numbers: the total angular momentum, $J$; the projection $K$ of $\mathbf{J}$ onto the molecular axis; and the projection $M_{J}$ of $\mathbf{J}$ onto a lab-frame axis, in this case the external field direction. The rotational motion is precessional: the molecular axis precesses about the total angular momentum vector $\mathbf{J}$, which in turn precesses about the lab-frame axis. Solving the equations of motion for a symmetric top molecule through a hexapole field (shown in Figure 3), yields simple harmonic oscillator solutions for the radial coordinate. Molecules in lower Stark states follow exponential trajectories that diverge from the hexapole axis, and are lost from the molecular beam. Conversely, upper Stark states follow sinusoidal trajectories, $r(t) \propto \sin (\omega t)$, which focus back to the axis at a distance that depends on the rotational state and the potential applied to the hexapole rods, as determined by the oscillation frequency, $\omega$.

$$
\omega=\left(\frac{6 V_{0} \mu}{m r_{0}^{3}} \frac{\left|K M_{J}\right|}{J(J+1)}\right)^{1 / 2}
$$

Here $V_{0}$ is the hexapole potential, $m$ and $\mu$ are the mass and dipole moment of the molecule, and $r_{0}$ is the inscribed radius of the hexapole rods. The dependence of $\omega$ on both $V_{0}$ and the rotational state of the molecule means that the hexapole potential can be chosen to focus any desired rotational state with $K M \neq 0$ onto the beam axis at the hexapole exit. Other non-focusing rotational states may be eliminated from the beam if desired by using an iris or pinhole to allow only the focused state of interest to exit the hexapole.

The upper Stark states transmitted through the hexapole are all aligned with their dipoles 
opposing the applied electric field, but are not yet oriented in space, since the hexapole field is inhomogeneous. In order to spatially orient the beam it is allowed to pass adiabatically into a region of weak homogeneous electric field maintained between a pair of parallel plates positioned directly after the hexapole. As the beam leaves the hexapole the molecular dipoles rotate in space to line up against the new homogeneous field and orientation is achieved. A homogeneous field of only around $2 \mathrm{~V} \mathrm{~cm}^{-1}$ or more is required in order to ensure that the upper Stark states remain well-defined and thereby to achieve some degree of molecular orientation [23]. However, as we shall see in Section 2.6.1, considerably stronger fields are often required in order to overome coupling of $\mathbf{J}$ to any non-zero nuclear spins in the molecule in order to achieve optimum orientation.

The degree of orientation is determined by the rotational states of the molecules in the beam. In the strong-field case, where coupling of $\mathbf{J}$ to the electric field is much stronger than coupling to any non-zero nuclear spins, the average angle between the electric field vector and the molecular dipole (i.e. the precession angle) for a given $\left|J K M_{J}\right\rangle$ state is given by

$$
\langle\cos \theta\rangle=\frac{K M_{J}}{J(J+1)}
$$

A detailed comparison between the hexapole technique and the Brute Force alignment method described in the previous section has been carried out by Bulthuis et al.[24].

When using the hexapole technique in stereodynamics studies, the relatively weak orientation field employed raises the concern that the anisotropic long range part of the intermolecular potential experienced as a collision partner is approached could potentially lead to reorientation of the prepared molecules prior to collision, thereby blurring any steric effects. However, a number of studies employing the hexapole orientation method have shown clear steric preferences for reaction at one end of a symmetric-top molecule over the other [25, 26, 27].

\subsection{Alignment using a strong laser field}

The approaches described so far have used static electric fields to generate oriented distributions of molecules. There are also a range of techniques that employ lasers to generate alignment and orientation. In this section we will consider ways in which a non-resonant laser field may be used to induce spatial polarisation (in the following Section we will explore the case in which the laser frequency is resonant with an optical transition in the atom or molecule under study). 
Normand et al. [28] were the first to demonstrate that an intense non-resonant linearly-polarised laser field could induce strong molecular alignment, in their case in an ensemble of CO molecules. A full theory to explain their observations was developed by Friedrich and Herschbach [29], and applies when the laser pulse is long compared with the rotational period of the molecule, the so called 'adiabatic alignment' regime. The underlying mechanism is very similar to that of brute force orientation, with the key difference being that because the electric field of the laser changes direction throughout each optical cycle on a timescale much faster than a permanent molecular dipole can align with the field, the interaction is instead with an induced dipole within the molecule. As such, it depends on the molecular polarisability rather than on a permanent dipole moment, with the molecules aligning so that the molecular axis possessing the highest polarisability lies along the electric field vector of the laser. The alignment only lasts for the duration of the laser pulse: molecules come into alignment as the laser intensity increases through the rising edge of the pulse, the alignment reaches a maximum at the peak of the pulse, with the molecules occupying fieldinduced pendular states similar to those generated in brute force alignment, and the alignment then decreases and eventually disappears throughout the falling edge of the pulse. As in the case of brute force alignment, the attainable alignment can be improved by state-selecting molecules in their lowest rotational states prior to applying the alignment laser pulse. Using this technique, Holmegaard et al. [30] have achieved one-dimensional alignments in iodobenzene of $\left\langle\cos ^{2} \theta\right\rangle=0.97$, where $\theta$ is the angle between the molecular alignment axis and the laser polarisation. By performing the experiments in the presence of a static electric field, it is also possible to induce orientation in strongly polar molecules $[30,31,32]$. These experiments will be considered further in Section 3.1.

A linearly polarised laser pulse allows molecules to be aligned along a single axis, but, as demonstrated by Stapelfeldt and coworkers [33], it is also possible to achieve alignment with respect to two or even three axes using a circularly or elliptically polarised laser field. This is one of very few techniques that effectively allow the rotational motion of the molecule to be eliminated in three dimensions. The alignment scheme has the appeal of being straightforward to implement and, in contrast to the orientation techniques described previously, is applicable to virtually any molecule. However, in common with brute force orientation, it does not allow experiments to be carried out on aligned molecules under field free conditions.

It has also been shown that laser pulses much shorter than the rotational period of the molecule 
can induce transient alignment [33]. This phenomenon is usually referred to as 'dynamical' or 'nonadiabatic' alignment in order to distinguish it from the adiabatic alignment described above. The ultrashort laser pulses required to achieve dynamical alignment have a broad frequency bandwidth, and excite a coherent superposition, or 'wavepacket', of excited rotational states of the molecule in its electronic and vibrational ground state. Once the laser pulse is turned off, the various components of the wavepacket evolve in time, dephasing and coming back into phase with each other periodically. On each 'rotational revival', when the components of the wavepacket are in phase, the molecules are spatially aligned. Since the revivals occur at predictable times, this provides a means of carrying out experiments on aligned molecules in a field free environment. Dynamical alignment has been explained and modelled comprehensively by Seideman [34].

\subsection{Alignment through polarised laser excitation}

All of the methods we have considered so far rely on modifying the behaviour of every molecule within the sample of interest. An alternative approach is simply to select molecules that have the desired polarisation from a randomly oriented sample. This may be achieved readily with a high degree of control through polarised laser excitation, as a consequence of the vector character of the transition dipole linking two quantum states within a molecule.

When an atom or molecule absorbs or emits a photon and undergoes a transition from an initial state $i$ to a final state $f$, the associated redistribution of charge may be represented by an electric dipole moment, or 'transition dipole', $\boldsymbol{\mu}_{i f}$. The transition dipole depends on the initial and final state wavefunctions, and on the electric dipole operator $\hat{\mathbf{d}}$ of the molecule.

$$
\boldsymbol{\mu}_{i f}=\left\langle\psi_{f}|\hat{\mathbf{d}}| \psi_{i}\right\rangle
$$

For an electric dipole transition to occur, the transition dipole must interact with the electric vector $\mathbf{E}$ of electromagnetic radiation of the appropriate frequency. The transition probability is then

$$
P_{i f} \propto\left|\boldsymbol{\mu}_{i f} \cdot \mathbf{E}\right|^{2}=\mu_{i f}^{2} E^{2} \cos ^{2} \theta
$$

where $\theta$ is the angle between $\boldsymbol{\mu}_{i f}$ and $\mathbf{E}$. The probability of a molecule absorbing a photon is therefore strongly dependent on the angle between the transition dipole and the electric field vector of the light. Now consider the case where linearly polarised light is incident on a randomly oriented 
sample of gas. The $\cos ^{2} \theta$ dependence in Equation (6) means that atoms or molecules oriented such that their transition dipoles are parallel to the electric vector have the highest probability of absorbing a photon, while those oriented with their transition dipoles at right angles to the electric vector have zero probability of absorbing a photon.

This behaviour may be exploited to prepare aligned distributions of either ground-state or excited-state molecules. This is most easily demonstrated by considering a sample of diatomic molecules. The most common types of transition in a diatomic are $\Sigma \leftarrow \Sigma$ transitions, for which the transition dipole lies parallel to the bond, and $\Sigma \leftarrow \Pi$ or $\Pi \leftarrow \Sigma$ transitions, for which the transition dipole lies perpendicular to the bond. Exciting a $\Sigma \leftarrow \Sigma$ transition with a linearly polarised laser will yield an aligned distribution of excited states with a $\cos ^{2} \theta$ distribution of bond axes about the laser polarisation direction. If the transition is saturated then the ground states left behind will be aligned with a $\sin ^{2} \theta$ distribution of bond axes about the laser polarisation direction. The situation would be reversed if we chose to excite on a perpendicular transition: the excited state would now exhibit a $\sin ^{2} \theta$ distribution of bond axes, and the ground state a $\cos ^{2} \theta$ distribution. When dealing with mixed transitions in a diatomic molecule, which have both parallel and perpendicular character, or when dealing with polyatomic molecules, the transition dipole has a more complicated relationship to the molecular geometry than in the simple examples given above. However, while the situation becomes more complex, the same principles still apply.

We should note that the alignment generated through polarised excitation will rapidly be degraded by molecular rotation, with higher rotational states randomising the distribution on a faster timescale than lower rotational states. However, as we shall see in Section 4, the polarised excitation approach is ideally suited for the fast timescale of laser pump-probe experiments, and has been used in a number of experiments to prepare aligned distributions of vibrationally or electronically excited molecules for a range of dynamical studies.

If the upper state is dissociative, then the alignment of the bond axes in the selected molecules is transformed into a polarised velocity distribution in the photofragments. This is illustrated in Figure 4, which shows the 3D velocity distribution of $\mathrm{Cl}$ atoms formed in the linearly polarised photolysis of $\mathrm{Cl}_{2}$ via the $C$ state at $308 \mathrm{~nm}$. Because the dissociation step involves a perpendicular transition, molecules oriented with their bond axes perpendicular to the electric vector of the laser beam are dissociated preferentially, leading to $a \sin ^{2} \theta$ distribution of photofragments about the 


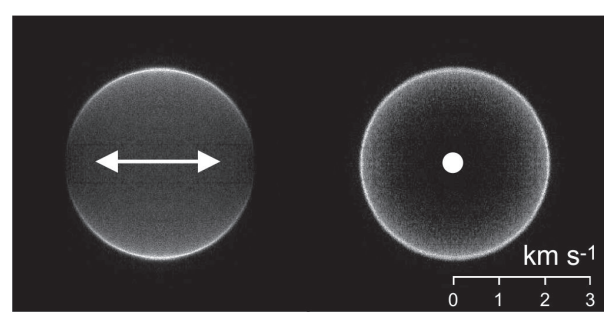

Figure 4: Velocity-map images of ground-state $\mathrm{Cl}$ atoms formed in the $308 \mathrm{~nm}$ photolysis of $\mathrm{Cl}_{2}$. Dissociation occurs via a pure perpendicular transition, and the images clearly show the resulting $\sin ^{2} \theta$ velocity distribution of the $\mathrm{Cl}$ atoms, with signal intensity peaking at right angles to the polarisation vector of the photolysis light (shown in white in the centre of the images). The atoms are detected by $(2+1)$ REMPI via the $4 p\left({ }^{4} P_{3 / 2}\right) \leftarrow{ }^{2} P_{3 / 2}$ transition.

laser polarisation vector.

As a small aside, it was quickly realised $[35,36]$ that such an approach provides a means for generating strongly anisotropic velocity distributions within a bulk gas-phase sample, effectively yielding a 'molecular beam in a bulb'. This led to the development of an alternative technique to crossed molecular beam scattering for carrying out reaction dynamics investigations. The Photoloc technique, as this approach was christened, allows reaction product velocity (angular and speed) distributions to be recorded without the need for high vacuum, and has yielded considerable insight into the dynamics of a wide range of chemical reactions.

\subsection{Alignment resulting from photofragmentation}

Many chemical processes yield products in which the electronic angular momentum is strongly polarised. As an example, atomic and molecular photofragments often exhibit strong polarisation relative to the direction of the breaking bond. This should not come as a surprise: the electronic angular momentum of an atomic or molecular fragment is determined by the distribution of unpaired electron density, which is often highly anisotropic during the bond dissociation process. If the bond axis of the dissociating parent molecule is polarised in the lab frame, for example by preparing the dissociating state through polarised laser excitation, as described in the previous section, then the photofragments will also be polarised in the lab frame. The velocity and bond-axis or angular momentum polarisation of the photofragments will depend both on the dissociation process itself, and on the polarisation of the photolysis laser. 
Polarisation arising from photodissociation has been a lively field of study for a number of years. The observed polarisation is often relatively modest, and rather than use the photodissociation process to generate polarised photofragments for further experiments, efforts have tended to be directed at measuring photofragment angular momentum polarisation in order to probe the dynamics of the dissociation process itself $[37,38]$. However, a few photodissociation systems have been discovered in which the degree of photofragment polarisation is extraordinarily strong, and which show considerable promise as a source of polarised atoms or radicals for scattering studies. Perhaps the strongest atomic polarisation seen to date is that noted in a number of studies $[39,44,45,46,47]$ of $\mathrm{O}\left({ }^{1} D\right)$ atoms produced in the photodissociation of molecular oxygen via the $B^{3} \Sigma_{u}^{-}$state. The transition from the ground state, $X^{3} \Sigma_{g}^{-}$, to the $B$ state is the first fully allowed spectroscopic transition in molecular oxygen, and is responsible for the Schumann-Runge bands and continuum observed in the VUV absorption spectrum of the earth's atmosphere. Regardless of whether the $B$ state is accessed by a one-photon transition or a two-photon transition, and virtually independently of wavelength over the range from threshold at $175 \mathrm{~nm}$ all the way down to $120 \mathrm{~nm}$, the $\mathrm{O}\left({ }^{1} D\right)$ fragment is formed almost exclusively in its $M_{J}=0$ quantum state, i.e. with $\mathbf{J}$ polarised in a direction perpendicular to the recoil velocity. The source of the polarisation in this system is particularly simple to understand, at least to first order, and provides a good example in the context of the present discussion.

We will assume the $B$ state to be a pure $\Sigma$ state with $\Lambda=\left|M_{L}\right|=0$, where the bond axis is the axis of quantisation. The state dissociates (transforming the bond axis of the parent $\mathrm{O}_{2}$ molecule onto the recoil axis of the atomic photofragments) to give an $\mathrm{O}\left({ }^{3} P\right)$ and an $\mathrm{O}\left({ }^{1} D\right)$ atom, which must either have $M_{L}\left({ }^{3} P\right)=0, M_{L}\left({ }^{1} D\right)=0$ or $M_{L}\left({ }^{3} P\right)= \pm 1, M_{L}\left({ }^{1} D\right)=\mp 1$. The major contribution to the $B$ state is from the former of these possibilities, and the $\mathrm{O}\left({ }^{1} D\right)$ therefore has $M_{L}=0$. By virtue of being a singlet state, it also has $M_{S}=0$, and therefore $M_{J}=0$ i.e. $\mathbf{J}$ is polarised perpendicular to the recoil axis, in accord with experiment.

While the experiments reveal a strong preference for $\mathrm{O}\left({ }^{1} D\right)$ formation in $M_{J}=0$, there is also a small amount of population in other $M_{J}$ states, primarily $M_{J}= \pm 1$. More comprehensive models of the dissociation dynamics which go some way towards explaining this observation are presented by $\mathrm{Wu}$ et al. [39] 


\subsection{Conditions for maintaining alignment and orientation}

Many of the techniques by which aligned and oriented molecular distributions can be prepared require the presence of an electric field of some description to define the polarisation axis. However, there are numerous instances when we might want to carry out experiments using a polarised molecular distribution but under field free conditions. This is especially true for experiments involving charged particles, such as photoelectron spectroscopy, electron diffraction, and studies of ion-molecule or electron-molecule collisions. For this reason it is important to understand the dependence of orientation or alignment on the strength of the orienting field, and also the mechanisms by which molecules deorient once the field is switched off, and the timescale over which this occurs.

\subsubsection{Orientation field}

Brute force orientation, adiabatic strong laser field alignment, and hexapole-based techniques all employ an electric field to achieve orientation. As we have already seen, brute force orientation, and the related technique of strong laser field alignment, requires extremely high electric fields in order to achieve orientation, on the order of $100 \mathrm{kV} \mathrm{cm}^{-1}$. In both of these methods, the field must be strong enough to 'lock' the permanent or induced dipole moment of the molecule to an axis. In contrast, once upper-Stark-state molecules have been state selected in a hexapole, orientation is achieved as soon as the strength of the uniform orientation field is sufficient to define a new labframe quantisation axis about which the molecules precess. A number of researchers have carried out experiments to determine the required field strength to achieve this. One of the most common techniques is to use a split hexapole with a region of uniform field in the centre, provided by a pair of parallel plates. The fields provided by the first and second hexapoles are known as the A and B fields, respectively, and the uniform field in the centre is known as the C field. After passing through the A field the beam consists predominantly of upper Stark states. If the C field is non-zero the molecules make adiabatic transitions from the A field to the $\mathrm{C}$ field, and from the $\mathrm{C}$ field to the B field. They remain in the same rotational state throughout their trajectories through the system and will nearly all be transmitted through the B field to the detector. Conversely, if the C field is zero, the Stark energy levels become degenerate, the $M$ quantum number is undefined, and the molecules in the beam can rotate freely as they travel through the parallel plates. If complete 
orientational scrambling occurs in this region then the molecules will have random orientations as they enter the B field, and the instantaneous orientation of any given molecule with respect to the field direction in the hexapole will determine whether it becomes an upper Stark state, lower Stark state, or $K M=0$ state in the B field. Upper Stark states are transmitted to the detector, but a significant fraction of the beam will be lost as non-focusing states, leading to a large drop in the beam signal relative to the case when the $\mathrm{C}$ field is non-zero. By monitoring the signal at the detector as the $\mathrm{C}$ field is increased, the field required to maintain orientation may be determined. Experiments of this type were first carried out by Brooks et al in 1969 [40], and more recently by Harland et al [23]. A representative set of data from the latter experiments is shown in Figure 5. In both cases modest fields of only a few volts per centimetre were found to be sufficient to ensure the required adiabatic transition from the hexapole field into the orienting field. This is consistent with the results of experiments in which steric effects were measured in photodissociation, chemical reactions, or photoelectron spectroscopy as a function of orientation field strength $[41,42,43]$.

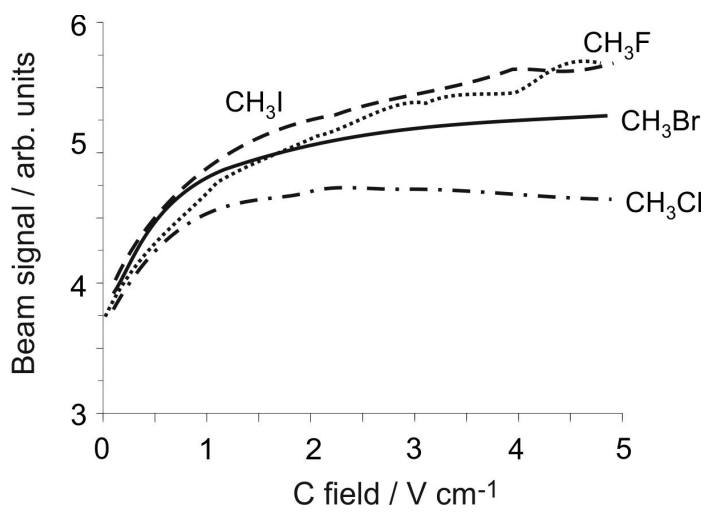

Figure 5: Measurement of the minimum field required to achieve orientation following hexapole state selection of symmetric top molecules. Molecules state-selected in a first hexapole will only focus in a second hexapole if the field strength in the intervening uniform $\mathrm{C}$ field is sufficient to maintain the distribution of Stark states.

It is clear that the upper Stark states selected by a hexapole may be oriented at very low electric field strengths. However, this is not a complete description of the situation. While such low fields are sufficient to maintain the integrity of upper Stark states, they are not sufficient to achieve optimum orientation. This is due to an additional angular momentum coupling, which we have not considered so far, namely hyperfine coupling between the rotational angular momentum $\mathbf{J}$ and the nuclear spin angular momentum of the molecule, I. Under weak-field conditions, the 
coupling between $\mathbf{J}$ and $\mathbf{I}$ is much stronger than the coupling of $\mathbf{J}$ to the electric field i.e. the Stark energy is much smaller than the hyperfine energy. The coupling between $\mathbf{J}$ and $\mathbf{I}$ splits each $\mid J K M_{J}>$ rotational state into a manifold of $\mid F J K I M_{F}>$. J now precesses about $\mathbf{F}$, and F precesses about the electric field. This reduces the degree of orientation over that obtainable for precession of a pure $\mid J K M_{J}>$ state about the field, with the reduction in orientation varying from molecule to molecule depending on its nuclear spin states. On moving to stronger fields, of the order of hundreds of $\mathrm{V} \mathrm{cm}^{-1}$, the Stark energy now greatly exceeds the hyperfine energy, and the precession of $\mathbf{J}$ around $\mathbf{E}$ is so fast that the nuclear orientation cannot respond on the same timescale. I and $\mathbf{J}$ are now decoupled, and optimum alignment is achieved.

This explains the results obtained by Janssen and coworkers [48] and described in Section 3.1, in which relatively high field strengths were required to achieve optimum orientation of $\mathrm{CD}_{3} \mathrm{I}$, a molecule with a large number of possible nuclear spin states. Similar results were observed in the photoionization experiments of Ghandi and Bernstein [42, 43]

\subsubsection{Deorientation}

The above discussion leads us neatly on to the topic of deorientation of a spatially polarised ensemble of atoms or molecules in the absence of an orienting field. Deorientation, also known as 'depolarisation' or 'spatial scrambling', occurs primarily through the hyperfine interaction. In any molecule containing one or more nuclei with non-zero nuclear spin, coupling of $\mathbf{J}$ to a manifold of nuclear spin states $\mathbf{I}$ rapidly leads to a random spatial distribution of $\mathbf{J}$ vectors. Since scrambling is mediated by the hyperfine interaction, we might expect the lifetime over which orientation can be maintained under field free conditions to be on the order of the hyperfine precessional period. This is indeed found to be the case. For example, the hyperfine frequencies for the methyl halides, of the order of $6 \mathrm{KHz}$, would lead us to predict orientation lifetimes of around $150 \mu \mathrm{s}$. This is in line with a variety of measurements, made either in a split hexapole setup by switching the C field to zero for increasing intervals, or by monitoring steric effects under field free conditions at varying times after preparing the oriented distribution [23]. 


\section{Characterisation of aligned and oriented atoms and molecules}

As noted in Section 1.2, we can consider atomic and molecular polarisation either in terms of polarisation of one or more bond axes, or in terms of polarisation of the total angular momentum. It should therefore come as no surprise that in order to characterise a spatially polarised distribution we can choose to measure either the spatial distribution of bond axes or of angular momentum vectors. Both approaches have been used, and will be explored in more detail in the following.

\subsection{Measuring the bond axis distribution}

The bond axis distribution in a sample of spatially oriented molecules is rather difficult to measure directly, but may be measured indirectly by photodissociating the bond of interest and measuring the recoil directions of the photofragments. The most common approach employs a laser pumpprobe scheme with velocity-map imaging detection. The pump laser performs the photodissociation, the probe laser ionizes the fragment of interest, and the resulting ions are mapped onto a positionsensitive detector by an electric field in order to measure their velocity distribution. This and similar approaches have been used to characterise spatially oriented samples prepared both by hexapole methods and by strong laser field alignment $[48,49]$. Here we will consider two examples.

Our first example concerns work carried out by Stapelfeldt and coworkers [30] on the iodobenzene molecule. Iodobenzene has a large polarisability along the axis containing the C-I bond, and in the presence of a linearly polarised Nd:YAG beam the molecules line up in pendular states with the C-I bonds parallel to the electric field vector of the light. The resulting spatial distribution of the molecules may be imaged by dissociating the C-I bond and recording the velocity distribution of the scattered iodine atoms. Rather than using a two-step laser pump-probe scheme to achieve this, Stapelfeldt and coworkers employed a single-step process in which an intense femtosecond laser pulse was used to strip all of the valence electrons from the molecule. In the resulting 'Coulomb explosion' the ionized atoms rapidly repel each other along the directions of the broken bonds, and by gating the position-sensitive detector to match the arrival time of the ionised iodine atoms, an image reflecting the spatial distribution of the C-I bonds is obtained. Detection via Coulomb explosion has the advantage that all molecules are dissociated with essentially equal probability, regardless of their orientation in space, so that the final image clearly reflects the distribution of 
orientations in the sample.

The effect of the alignment laser pulse is seen clearly in Figure 6. The two rings visible in the images are a result of two-photon and three-photon Coulomb explosion processes in which iodobenzene fragments to give $\mathrm{I}^{+}+\mathrm{C}_{6} \mathrm{H}_{5}^{+}$(innermost ring) and $\mathrm{I}^{+}+\mathrm{C}_{6} \mathrm{H}_{5}^{2+}$ (outermost ring), respectively. Panel (a) shows an image recorded with the alignment laser pulse turned off, and we see that there is no preferred direction in which the iodine atoms fly following dissociation. In contrast, panel (b) shows an image in which the Coulomb explosion was timed to match the peak of the alignment laser pulse. The alignment pulse was polarised vertically, from top to bottom in the image, and we see that iodine atoms are produced in a tight cone about this direction, indicating that the C-I bonds in the iodobenzene molecules were exceptionally strongly polarised along the laser polarisation axis. The alignment can be improved still further by selecting low rotational states from the molecular beam prior to the laser alignment step. This was achieved using an electrostatic deflector that deflected high-field-seeking states upwards, with the extent of the deflection depending on the rotational state. Different rotational states could then be probed by overlapping the alignment and probe laser beams in space with the desired state. Panels (c) and (d) of Figure 6 show the alignment distributions arising from the lowest and highest rotational states, respectively. If the polarisation of the alignment laser is moved out of the image plane, it is found that the combined effect of the alignment laser field and the high voltage applied along the time-of-flight axis to extract the ions to the detector results not only in molecular alignment, but also orientation of the molecular dipole in the static electric field.

Our second example concerns the characterisation by Janssen and coworkers [48] of a distribution of oriented $\mathrm{CD}_{3} \mathrm{I}$ molecules. A hexapole was used to select molecules in the $|J K M\rangle=|111\rangle$ state, which were then oriented in a uniform electric field. The oriented molecules were dissociated by a $266 \mathrm{~nm}$ laser pulse, and atomic iodine fragments in the spin-orbit excited ${ }^{2} P_{1 / 2}$ state were ionized by $(2+1)$ REMPI at around $305 \mathrm{~nm}$ and velocity-map imaged. Images were recorded as a function of the orienting field strength in order to investigate the field strength dependence of the orientation achieved. Images at three different field strengths are shown in Figure 7.

The laser used to photolyse the $\mathrm{CD}_{3} \mathrm{I}$ molecules was polarised vertically relative to the image. The transition dipole for the photolysis step lies along the C-I bond, and in the absence of any molecular orientation we would therefore expect to see $\cos ^{2} \theta$ distribution of atomic iodine 


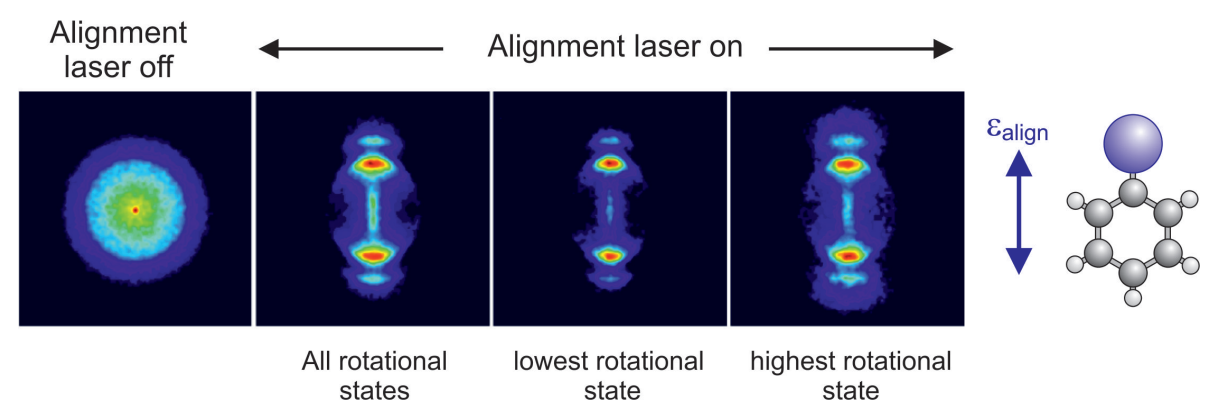

Figure 6: Laser alignment of iodobenzene molecules, detected through velocity-map imaging of I atoms produced when the molecule is Coulomb exploded by a high intensity femtosecond probe pulse. In (a) the alignment laser is turned off, and the distribution of I atoms is isotropic. In (b)-(d) the alignment laser is turned on, and the iodobenzene is strongly aligned, more so for low rotational states (image (c)) than high rotational states (image (d)). The alignment laser polarisation $\epsilon_{\text {align }}$ is indicated to the right of the images. Adapted from reference [30].

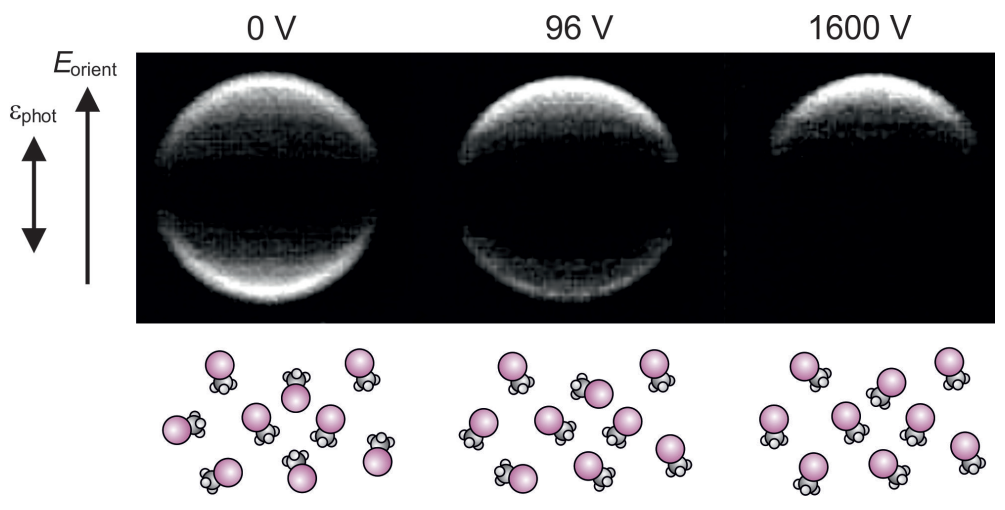

Figure 7: Velocity-map images recorded for I* fragments following $266 \mathrm{~nm}$ dissociation of hexapole stateselected and oriented $\mathrm{CD}_{3} \mathrm{I}$ in its $|J K M\rangle=|111\rangle$ state. The direction of the orienting field and photolysis laser polarisation are shown to the left of the images, and the images recorded from left to right illustrate the effect of increasing the orienting field over the range from 0 to $1600 \mathrm{~V} \mathrm{~cm}^{-1}$. Adapted from reference [48].

photofragments (see Section 2.4). This is precisely what we do see in the image shown in Figure 7 (a), recorded with an orienting field of zero such that the $\mathrm{CD}_{3} \mathrm{I}$ molecules were state selected but not oriented. In images (b) and (c) an orienting field is applied such that the molecules start to align with the iodine end uppermost. As the orienting field is increased, we first see a slight increase in the number of I atoms flying in the upwards direction relative to the downwards direction, and then at higher field strengths, when orientation is complete, we see only intensity at the top of the image, indicating that there are no molecules in the sample with the iodine atom pointing 
down. There are two points to note. Firstly, while the intensity distribution in the images depends strongly on the orientation distribution of the molecules, there is also a $\cos ^{2} \theta$ weighting that reflects the probability of photolysis as a function of the angle between the C-I bond and the polarisation axis of the photolysis laser. Secondly, the degree of orientation observed is considerably less than that seen in our previous example of iodobenzene oriented in a strong laser field. This reflects the selected rotational state of the molecules. The average angle to the $z$ axis for a state $|J K M\rangle$ is given by Equation (4), which for the $|111\rangle$ state yields an angle of $60^{\circ}$. To achieve more tightly oriented molecular distributions we would need to select states with higher values of $J$ and $M$. For example, the $|222\rangle$ and $|333\rangle$ rotational states have most probable angles of $48.2^{\circ}$ and $41.4^{\circ}$, respectively.

Interestingly, the field strengths required by Janssen and coworkers to achieve orientation of $\mathrm{CD}_{3} \mathrm{I}$ are considerably higher than those found in similar hexapole state selection and orientation experiments performed by a number of other researchers. This issue will be explored more thoroughly in Section 2.6.1.

\subsection{Measuring the angular momentum polarisation}

In Section 2.4 we noted that the transition probability for a spectroscopic transition depends on the angle between the polarisation vector of the light and the molecular transition dipole (Equation (6)). As a consequence, exciting a spectroscopic transition using a polarised laser can be used as a means of selecting an aligned subset of molecules. We can exploit the same ideas to measure atomic or molecular angular momentum polarisation, by noting that the transition dipole $\boldsymbol{\mu}_{i f}$ for any chosen detection transition is correlated with the total angular momentum vector, J. For example, a 'Q branch' transition, for which $\Delta J=0$, has $\boldsymbol{\mu}_{i f}$ parallel to $\mathbf{J}$, while a $\mathrm{P}$ or $\mathrm{R}$ branch transition, with $\Delta J= \pm 1$, has $\boldsymbol{\mu}_{i f}$ perpendicular to $\mathbf{J}$. If the angular momentum vectors, and therefore the transition dipoles, are randomly oriented, then excitation with light of any polarisation will give rise to an identical signal. However, if the angular momentum distribution (and therefore the spatial distribution of transition dipoles) is polarised, then light of different polarisations will be absorbed to a different extent, depending on the number of transition dipoles with which it interacts. Angular momentum alignment may be detected via differential absorption of light linearly polarised along two orthogonal axes, while angular momentum orientation leads to differential absorption of left 
and right circularly polarised light.

Polarised laser detection techniques of this type are usually used as the probe step of a laser pump-probe sequence to characterise angular momentum polarisation in the products of a photodissociation or bimolecular reaction process. As such, they need to be sensitive to the product velocity as well as to its angular momentum polarisation. The two most widely used techniques are Doppler-resolved laser-induced fluorescence (LIF), and resonance-enhanced multiphoton ionization (REMPI), combined either with core-extraction time-of-flight or velocity-map imaging detection. A single Doppler-resolved LIF measurement yields a one-dimensional projection of the product velocity distribution onto the laser propagation axis. Two or more projections are needed in order to reconstruct the full $3 \mathrm{D}$ velocity distribution, which means making measurements in which the probe laser propagates along different axes, and in each case employing the appropriate polarisations to measure the angular momentum polarisation. As an example, Figure 8 shows a series of Doppler-resolved LIF profiles recorded for quantum-state-selected CO products formed in the photolysis of glyoxal [50]. Two possible dissociation mechanisms for glyoxal are shown in Figure 8(a), one which leads to the rotational angular momentum $\mathbf{J}$ of the CO fragment becoming aligned parallel to its velocity vector, and one which leads to the two vectors being mutually perpendicular. These two mechanisms can be distinguished on the basis of the Doppler profiles. As shown in Figure 8(b), CO products with a large velocity component along the laser propagation axis are detected towards the wings of the Doppler profiles, while those travelling perpendicular to the laser propagation direction are detected near the centre of the Doppler profiles. Taking the Q-branch transition as an example, we see a dip in the centre of the Doppler profile, indicating that CO molecules travelling perpendicular to the laser propagation axis (or, equivalently, parallel to the laser polarisation vector) are detected less efficiently than those travelling parallel to the axis (perpendicular to the polarisation vector). These observations imply that the transition dipole $\mu$ for the probe step lies preferentially perpendicular to the product velocity vector. For products detected on a Q-branch transition $(\mu \| \mathbf{J})$, it follows that $\mathbf{J}$ is also perpendicular to the velocity, and that the CO fragment therefore undergoes a 'frisbee-type' motion as it departs from the interaction region. Similar arguments applied to the P and R branch Doppler profiles lead to the same conclusion.

REMPI combined with core-extraction time-of-flight detection also provides a one-dimensional projection of the velocity and polarisation distribution, and experiments are carried out in much 
(a)
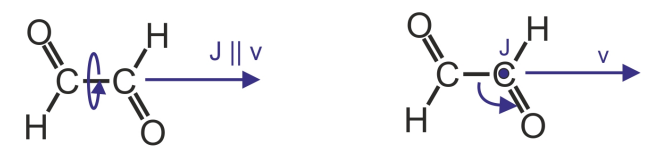

(b)

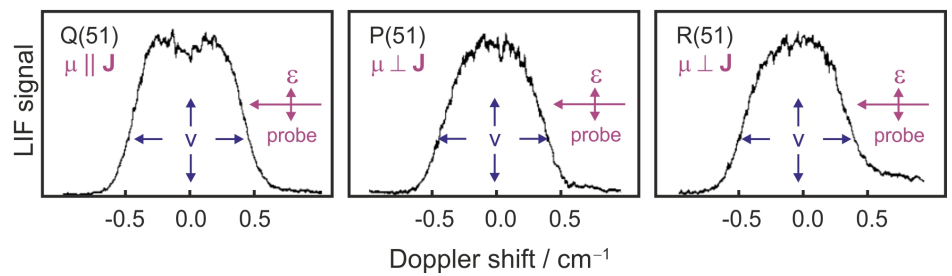

Figure 8: (a)Proposed mechanisms for glyoxal dissociation leading to $\mathbf{J} \| \mathbf{v}$ (left) and $\mathbf{J} \perp \mathbf{v}$ (right); (b) Doppler profiles for $\mathrm{CO}$ fragments formed in $(v=0, J=51)$. As explained in the text, the characteristic peak shapes observed for the $\mathrm{P}, \mathrm{Q}$, and R transitions allow the two mechanisms to be distinguished. Adapted from reference [50].

the same way as for Doppler-resolved LIF. These two techniques have been applied to the characterisation of photofragment and reaction product polarisation in numerous chemical systems [51, 52], but have now largely been superseded by imaging techniques.

Velocity-map imaging yields a two-dimensional projection of the product scattering distribution, and as a result, fewer projections are generally needed in order to reconstruct the complete product scattering distribution. In fact, a great deal of qualitative information on angular momentum polarisation can often be obtained from visual inspection of a single image. We will demonstrate this by continuing with our example of the VUV photolysis of $\mathrm{O}_{2}$ from Section 2.5, in which the $\mathrm{O}\left({ }^{1} D\right)$ photofragments are formed with $\mathbf{J}$ strongly polarised perpendicular to the recoil velocity. Figure 9 shows a series of velocity-map images of recoiling $\mathrm{O}\left({ }^{1} D\right)$ atoms, recorded using two different experimental geometries defined by the photolysis and probe laser propagation and polarisation axes. The pump and probe laser beams crossed at right angles in the image plane, and the geometries are further described by $\mathrm{H}$ and $\mathrm{V}$ labels for the photolysis and probe laser beams, denoting polarisation of the relevant laser in the plane of the image $(\mathrm{H})$ or normal to the plane of the image $(\mathrm{V})$, respectively.

In all of the images, the photolysis laser is polarised in the vertical direction relative to the images on the page. Since the transition to the $B$ state is parallel in character, parent $\mathrm{O}_{2}$ molecules in the randomly oriented molecular beam whose bond axis has an appreciable component in this direction will be preferentially dissociated, and we therefore expect maximum intensity at the top 


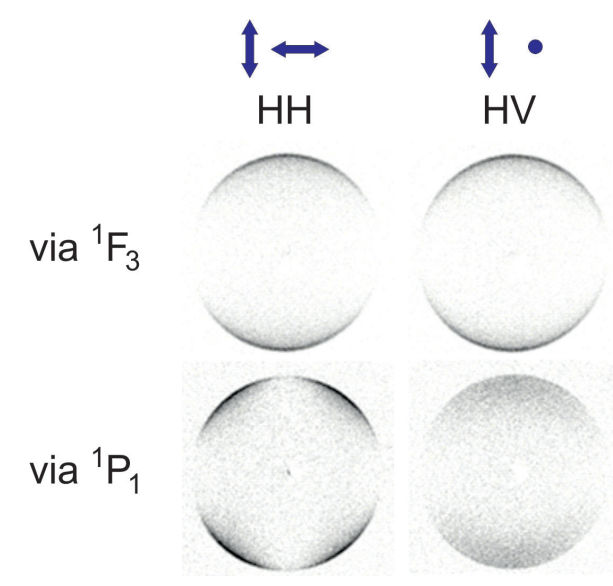

Figure 9: Velocity-map images of $\mathrm{O}\left({ }^{1} D\right)$ from the photodissociation of $\mathrm{O}_{2}$ at $157 \mathrm{~nm}$. As described in the text, images were acquired on two REMPI transitions using two experimental geometries defined by the pump and probe laser propagation and polarisation axes.

and bottom of the images. This is clearly seen to be the case in the HV geometry. As noted previously, the photodissociation dynamics yield $\mathrm{O}\left({ }^{1} D\right)$ atoms whose angular momentum (and therefore transition dipole for the probe step) is strongly aligned in a direction perpendicular to the photofragment velocity. We would therefore expect the detection efficiency with a laser of fixed polarisation to depend on the scattering angle, and this is precisely what is observed. To illustrate this, consider the crushed image recorded in the $\mathrm{HH}$ geometry via the ${ }^{1} P_{1}$ state. For this probe transition, detection sensitivity is highest when $\mathbf{J}$ is perpendicular to the polarisation vector of the probe light; in the image this would correspond to fragments with $\mathbf{J}$ polarised vertically . The extreme top and bottom of the image corresponds to products recoiling in the vertical direction (perpendicular to the probe polarisation vector), and the almost complete lack of signal in these regions of the image indicates that $J$ is very strongly polarised at right angles to the velocity vector, as we know to be the case.

\section{Applications of aligned and oriented atoms and molecules}

The original motivation for preparing aligned and oriented molecules came from within the reaction dynamics community, stemming from the desire to study chemical reactions under single collision conditions with full control over molecular orientation. The ability to prepare spatially aligned and oriented distributions of molecules in the gas phase has opened the way to detailed investiga- 
tions into steric effects in many different chemical processes. Gas-phase neutral and ion-molecule reactions, inelastic scattering, surface scattering, and a range of ionization processes have all been studied as a function of molecular orientation. In the following we will not attempt to provide a comprehensive overview of every experiment that has ever used spatially polarised species. Instead, we will focus on a number of selected examples, with the aim being to provide the reader with an appreciation of the possibilities.

\subsection{Inelastic and reactive scattering}

'Steric effects' are one of the most fundamental concepts in chemical kinetics and dynamics, yet there are very few ways in which such effects may be studied directly. It is therefore not too surprising that oriented-molecular-beam scattering techniques have been so enthusiastically adopted by the reaction dynamics community. Crossed-molecular -beam experiments were first carried out in the mid 1950s, and it was not until the 1960s that Dudley Herschbach and Yuan Lee began the work that would lead to their 1986 Nobel Prize in Chemistry. It is therefore rather remarkable that the first crossed beam reactive scattering measurements employing spatially oriented molecules were reported as early as 1966, by Phil Brooks of Rice University [25]. Brooks studied the reaction between atomic potassium and hexapole state-selected and oriented $\mathrm{CH}_{3} \mathrm{I}$, showing unequivocably that the iodine end of the molecule was more reactive than the methyl end. The steric factor, defined as $S=\left(S_{-}-S_{+}\right) /\left(S_{-}+S_{+}\right)$, with $S_{+}$and $S_{-}$the KI product signals arising from reaction at the positive and negative ends of the dipole, respectively, was found to be $0.6 \pm 0.3$. When the rather broad distribution of orientations within the molecular beam is taken into account, this suggests that a direct collision with the iodine end of the molecule is necessary for reaction. Since these early experiments, the hexapole technique has been and continues to be used with great success to study the stereochemistry of a large number of reactive and inelastic scattering systems involving symmetric top or pseudo-symmetric top molecules. For further examples, readers are referred to a recent review article [53].

A high degree of control was also achieved relatively early on over the polarisation of atomic reactants. In 1981, Rettner and Zare [54] used polarised laser excitation to prepare a molecular beam of calcium atoms in their electronically excited ${ }^{1} P_{1}$ state, in order to study the chemiluminescent 
reaction

$$
\mathrm{Ca}\left({ }^{1} P_{1}\right)+\mathrm{HCl} \rightarrow \mathrm{CaCl}\left(A^{2} \Pi, B^{2} \Sigma^{+}\right)+\mathrm{H} .
$$

By switching the polarisation of the excitation laser beam, the Ca atoms could be prepared so that their occupied $p$ orbital pointed either along or perpendicular to the reactant relative velocity vector. It was found that the first geometry preferentially formed $\mathrm{CaCl}$ in the $B^{2} \Sigma^{+}$state, while the second preferentially formed the $A^{2} \Pi$ state. This was interpreted in terms of the $p$ atomic orbital of the Ca atom evolving into either the $p \sigma$ or $p \pi$ molecular orbital of the excited state $\mathrm{CaCl}$ product.

Jumping to the present for our final scattering example, there has recently been a great deal of interest in studying interactions of polarised molecules with surfaces [55]. The desire to understand the dynamics of surface reactions in ever more detail is largely driven by their importance in a wide range of industrial processes, ranging from heterogeneous catalysis to chemical vapour deposition. A key model system for dynamics studies has been the interaction of $\mathrm{CH}_{4}$ with a nickel surface. Molecular beam experiments have demonstrated that dissociative chemisorption is an activated process that is favoured both by translational [56] and vibrational [57, 58, 59,60,61] excitation of the $\mathrm{CH}_{4}$. Recently, Beck and coworkers [62] have observed that adsorption is also strongly dependent on the alignment of the vibrationally excited $\mathrm{CH}_{4}$. In their experiments, a randomly oriented beam of $\mathrm{CH}_{4}$ was intercepted by a high power $\mathrm{CW}$ infrared laser tuned to excite one quantum of the $\mathrm{CH} \nu_{3}$ stretching vibration, before impinging on a clean $\mathrm{Ni}(100)$ surface. The laser was linearly polarised either parallel to or perpendicular to the surface, and the sticking probability was measured by detecting bound carbon atoms via Auger electron spectroscopy. $\mathrm{CH}_{4}$ molecules in their vibrational ground state did not give rise to a measurable signal, making the experiment sensitive purely to reaction of the stretch-excited $\mathrm{CH}_{4}$. The reaction probability with the surface was found to be up to $60 \%$ higher when the laser was polarised parallel to the surface than when it was perpendicular, indicating a strong alignment effect. The difference in reactivity could in principle be due to alignment either of the vibrational transition dipole, $\boldsymbol{\mu}_{i f}$ or the rotational angular momentum J of the rovibrationally excited $\mathrm{CH}_{4}$. However, the largest alignment effect was seen when $\mathrm{CH}_{4}$ was excited via the $R(0)$ line of the $\nu_{3}$ vibrational band. This yields a rotationless state with a spatially isotropic wavefunction, indicating that it is alignment of the stretching vibration (and not 
J) relative to the surface that controls the dissociation probability of $\mathrm{CH}_{4}$. The dependence of the reaction rate on vibrational orientation could arise either from the dissociation probability itself being alignment-dependent, or from the rate of vibrational energy transfer to the surface being alignment dependent. Which of these mechanisms is the correct one is still an open question.

\subsection{Electron impact ionization}

Electron impact ionization is widely used as an ionization technique in mass spectrometry, and is also an important process in plasmas and many other applications involving ionizing radiation, including medical physics and materials science. An incoming electron with an energy higher than the ionization threshold of the molecule is able to ionize the molecule, yielding a molecular ion and two outgoing electrons. At sufficiently high incident electron energies the molecular ion may fragment to form various daughter ionic and neutral species. Investigations into electron impact ionization of oriented molecules has shown that the process is strongly orientation dependent.

Harland and coworkers have studied the orientation dependence of the electron impact process for $\mathrm{CH}_{3} \mathrm{Cl}$, along with several other symmetric top molecules [26, 27]. An oriented beam of $\mathrm{CH}_{3} \mathrm{Cl}$ was prepared using the hexapole method and crossed at right angles with an electron beam. The resulting ions were detected by a quadrupole mass spectrometer. The distribution of orientations within the molecular beam was relatively broad, and for each polarity of the orienting field included a substantial fraction of molecules that could best be described as 'sideways oriented'. Nonetheless, a very strong steric effect was observed. At an electron energy of $200 \mathrm{eV}$, production of the molecular ion, $\mathrm{CH}_{3} \mathrm{Cl}^{+}$, was found to be 2.6 times more likely when the electron was incident on the positive $\left(\mathrm{CH}_{3}\right)$ end of the molecular dipole than on the negative $(\mathrm{Cl})$ end. The steric effect has been reproduced by several theoretical models [27, 63, 64], and may be interpreted in terms of the more attractive nature of the interaction potential between the electron and molecule when the negatively charged electron interacts with the positively charged end of the molecular dipole. Interestingly, at lower electron energies the steric effect is reduced. This has been interpreted as arising from the increasing de Broglie wavelength of the electron at lower energies. Once the de Broglie wavelength exceeds the dimensions of the molecule, the spatial resolution of the electron is insufficient to discriminate between the two ends of the molecular dipole.

The primary ionic fragmentation product of $\mathrm{CH}_{3} \mathrm{Cl}$ following electron impact ionization is the 
$\mathrm{CH}_{3}^{+}$ion, for which no steric effect was observed. Recalling that there will be roughly equal numbers of 'sideways oriented' molecules in the molecular beam, regardless of the nominal polarity of the orientation, this implies that fragmentation arises from 'broadside' collisions in which the electron collides with the $\mathrm{C}-\mathrm{Cl}$ bond.

\subsection{Photoionization and photoelectron imaging}

Photoelectron spectroscopy is widely used to probe the energy levels and electronic structure of atoms, molecules, and surfaces. Photoelectron imaging, in which the angular distribution of the photoelectrons is measured in tandem with their kinetic energies, provides further information on the structure and evolution of atomic and molecular orbitals within the system under study, and is an area of considerable current interest. Photoelectron imaging has been used as a detailed probe of the ionization process itself $[65,66,67,68,69]$, and also to study phenomena such as chirality [70], rotational wavepacket dynamics [71] and coherent control [72].

Photoionization experiments generally use either laser or synchrotron light. The photoelectron angular distribution (PAD) is the result of the ionized electron scattering from the ion core of the ionized atom or molecule. The form of the PAD depends strongly on the interaction between vector properties of the molecule under study (transition dipole moments, bond directions etc) and the polarisation vector of the ionizing radiation. Molecular alignment and orientation can therefore play an important role.

To understand the form of a PAD, we need to consider the wavefunction of the departing photoelectron. The wavefunction can be expressed as an expansion in spherical harmonics [73].

$$
\psi_{e}=\sum_{l, m} c_{l m} e^{i \delta_{l}} Y_{l m}(\theta, \phi)
$$

where the $c_{l m}$ coefficients contain radial and angular information on the initial and final state of the system, and $\delta_{l}$ is a scattering-induced phase shift. The spherical harmonics, or "partial waves' are defined by an orbital angular momentum quantum number $l$ and a lab-frame projection quantum number $m$. The number of terms in the expansion depends on the system under study, but is generally small due to the selection rules governing the photoionization process. For example, ionization from an atomic orbital with angular momentum $l$ gives rise to only two partial waves, 
with angular momenta $l-1$ and $l+1$. In molecules, $l$ is no longer a good quantum number and the situation becomes somewhat more complex, but the number of terms remains small.

The PAD is given by the coherent square of the wavefunction.

$$
I(\theta, \phi) \propto \psi_{e}^{*} \psi_{e}=\sum_{l m} \sum_{l^{\prime} m^{\prime}} c_{l^{\prime} m^{\prime}}^{*} c_{l m} e^{i\left(\delta_{l}-\delta_{l}^{\prime}\right)} Y_{l^{\prime} m^{\prime}}^{*}(\theta, \phi) Y_{l m}(\theta, \phi)
$$

which may be written in simplified form as

$$
I(\theta, \phi)=\sum_{L, M} B_{L M} Y_{L M}(\theta, \phi)
$$

with $\left|l-l^{\prime}\right|<<L<<l+l^{\prime}$ and $M=m+m^{\prime}$. The $B_{L M}$ coefficients contain all of the information on the photoionization dynamics, and depend upon the orbital from which the electron is ejected, the photoionization energy, the laser polarisations, and the distribution of vectors within the ionized molecule. As we shall see in the following, the ability to carry out photoelectron imaging experiments on oriented and aligned molecules greatly increases the amount of information available by increasing the number of $B_{L M}$ coefficients that may be extracted from the data.

When a single photon photoionization measurement is carried out on a randomly oriented sample of molecules, the experiment averages over all possible relative orientations of the laser polarisation vector and vectors within the molecule. The resulting PAD is cylindrically symmetric about the polarisation vector, and takes the form ${ }^{1}$

$$
I(\theta) \propto 1+B_{2} P_{2}(\cos \theta)
$$

In such an experiment we only obtain information on a single coefficient, $B_{2}$, characterising the scattering distribution. In contrast, if we could carry out an experiment on a perfectly spatially oriented molecule, the measured distribution would take the form

$$
I(\theta, \phi) \propto \sum_{L=0}^{L_{\max }} \sum_{M=-L}^{L} B_{L M} Y_{L M}(\theta, \phi)
$$

where $\theta$ and $\phi$ are measured relative to the molecular $z$ axis, and the sum terminates at $L_{\max }=$ $2 l_{\max }$, with $l_{\max }$ the highest orbital angular momentum quantum number of the generated partial

\footnotetext{
${ }^{1}$ Note that in the case of chiral molecules, the relevant expression is $I(\theta) \propto 1+B_{1} P_{1}(\cos \theta)+B_{2} P_{2}(\cos \theta)$.
} 
waves. We now have access to many more molecular frame $B_{L M}$ parameters, each of which provide insight into the electron-ion scattering potential.

An intermediate situation is that in which a randomly oriented sample is ionised by resonant multiphoton ionization. In this case the resonant excitation step selects a subset of molecules whose transition dipoles overlap with the laser polarisation vector (see Section 2.4), and it is this partially aligned or oriented distribution that undergoes ionization by a further photon. In this case, for an ionization involving $n$ photons to reach the resonant step and $m$ photons to ionize from this step (i.e. an $(n+m)$ REMPI process), the PAD takes the form

$$
I(\theta, \phi) \propto \sum_{L=0}^{2 n+2 m} \sum_{M=-L}^{L} B_{L M} Y_{L M}(\theta, \phi)
$$

with $L$ even and terms with $2 n+2 m>2 l_{\max }$ or $M>2 n$ equal to zero. In this case the $B_{L M}$ parameters depend on the molecular alignment prepared by the excitation beam, and in contrast to the two cases considered above, the PAD will reflect the photoionization dynamics of the resonant state rather than the ground state. Unlike in the case of a perfectly oriented molecule, information on odd values of $L$ is not available.

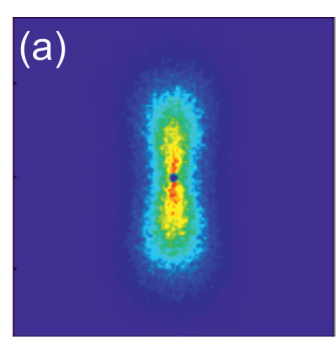

Random

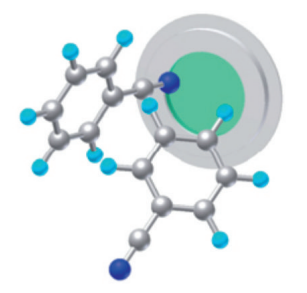

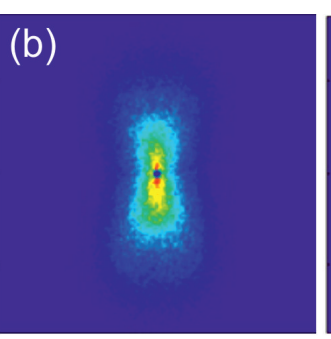

1D orientation

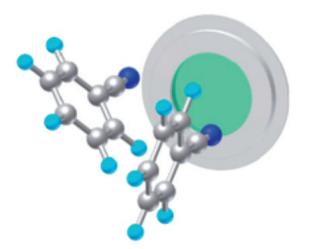

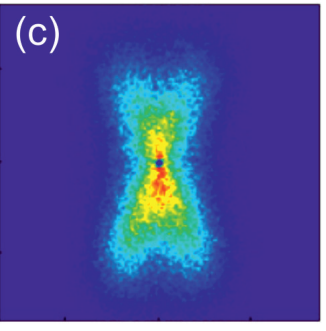

3D orientation

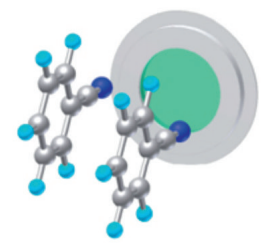

Figure 10: Photoelectron distributions recorded from benzonitrile molecules that are: (a) randomly oriented; (b) oriented in one dimension; and (c) oriented in three dimensions. In the case of three dimensional orientation the nodal plane of the molecular orbital in the plane of the molecule is revealed. Adapted from reference [74].

A beautiful example of the effect of increasing molecular orientation on the form of the PADs 
obtained on photoionization has recently been provided by Holmegaard et al. [74]. Figure 10 shows photoelectron angular distributions recorded from benzonitrile molecules that were: (a) randomly oriented; (b) oriented in one dimension by a high intensity linearly polarised laser pulse; and (c) oriented in three dimensions using a high intensity elliptically polarised laser pulse. Each incremental increase in the extent of molecular polarisation leads to dramatic changes in the measured PADs. The most striking feature is seen in the PAD from fully oriented molecules. The molecular plane of the benzonitrile coincides with the nodal plane of the two highest occupied molecular orbitals (the HOMO and HOMO-1). We would therefore expect electron emission to be strongly suppressed in the plane of the molecule, and this is clearly visible in the photoelectron images as a reduction in intensity in the 'up' and 'down' directions. As seen in the images from randomly oriented and onedimensionally oriented molecules, without the ability to orient the molecules completely in three dimensions, this feature is 'smeared out' through averaging over the different molecular orientations present in the sample.

Such experiments hint at the exciting possibility of future time-resolved experiments in which it will become possible to map out the structure and evolution of valence molecular orbitals in real time during a chemical reaction. Another area which is currently attracting considerable attention is time-resolved photoelectron holography $[75,76]$. This relies on recording interference patterns between a 'reference wave', part of the photoelectron wavefunction which is emitted from the atom or molecule without interacting with it, and a 'signal wave', the remainder of the wavefunction, which scatters from the target and encodes its structure. In order to achieve sufficient signal intensity, a large number of such 'single molecule holograms' must be superimposed, making a high degree of molecular orientation essential. While currently at a relatively early stage of development, such approaches have the potential to revolutionise chemical structure determination, essentially providing a gas-phase analogue for X-ray crystal structure determination methods.

\subsection{Larmor precession}

For our final example, we move to a rather different application of atomic alignment. Larmor precession of a quantum mechanical angular momentum vector about an applied magnetic field forms the basis for a range of magnetic resonance techniques, including nuclear magnetic resonance spectroscopy, electron spin resonance spectroscopy, magnetic resonance imaging, and quantum beat 
spectroscopy. Such techniques now represent the state of the art in medical imaging and in chemical structure determination and quantification. While not contributing directly to these techniques, the ability to prepare highly aligned distributions of atomic angular momenta has recently allowed the Larmor precession to be imaged for the first time [44].

As noted in Section 2.5, photolysis of molecular oxygen at VUV wavelengths yields $\mathrm{O}\left({ }^{1} D_{2}\right)$ atoms formed almost exclusively with $M_{J}=0$. These strongly aligned $\mathrm{O}\left({ }^{1} D_{2}\right)$ atoms turn out to provide a uniquely clean and simple model system for exploring Larmor precession. They have a long lifetime of around $110 \mathrm{~s}$, since emission of a photon to return to the ground state is spin forbidden. They have zero spin, so the angular momentum is purely orbital in character, i.e. the total electronic angular momentum $J$ is equal to the orbital angular momentum $L$, and also $M_{J}=M_{L}$. Finally, the overwhelmingly predominant ${ }^{16} \mathrm{O}$ isotope of oxygen has no nuclear spin, so there is no hyperfine depolarisation (see Section 2.6.2).

Larmor precession occurs when a magnetic moment associated with a quantum mechanical angular momentum interacts with a magnetic field. For a spin-zero system such as $\mathrm{O}\left({ }^{1} D_{2}\right)$, the magnetic moment $\boldsymbol{\mu}$ is related to the angular momentum $\mathbf{J}$ by

$$
\boldsymbol{\mu}=-\frac{e}{2 m_{e}} \mathbf{J}
$$

where $e$ and $m_{e}$ are the electronic charge and mass, respectively. When an external magnetic field $\mathbf{B}$ is applied to the system, the magnetic moment experiences a torque, $\boldsymbol{\tau}=\boldsymbol{\mu} \times \mathbf{B}$, perpendicular to the magnetic moment (and therefore perpendicular to $\mathbf{J}$ ), which causes the magnetic moment to precess about the field direction at the Larmor frequency $\nu_{\mathrm{L}}$.

$$
\nu_{L}=\frac{\mu_{B} B}{h}
$$

where $\mu_{B}=e \hbar / 2 m_{e}$ is the electronic Bohr magneton.

In Section 3.2 and Figure 9 we characterised the angular momentum distribution of the $\mathrm{O}\left({ }^{1} D_{2}\right)$ atoms formed in the VUV photolysis of $\mathrm{O}_{2}$. If the same experiments are carried out in the presence of a magnetic field, the $\mathrm{O}\left({ }^{1} D\right)$ angular momentum distribution will precess about the field during the time interval between the pump and probe lasers, and by recording images as a function either of the pump-probe delay or the magnetic field strength, the precessional motion may be 
visualised. Experimentally, this was achieved by installing Helmholtz coils to produce magnetic fields along the $x, y$, and $z /$ TOF axes of the velocity-map imaging apparatus. A representative set of images recorded as a function of magnetic field strength, with the magnetic field applied along the time-of-flight axis, is shown in Figure 11. At non-zero field strengths, the angular momentum distribution of the $\mathrm{O}\left({ }^{1} D\right)$ atoms formed in the photolysis step precesses about the field axis at a Larmor frequency determined by the magnetic field strength. Because the transition dipole moment for the probe step is correlated with the total angular momentum vector, $\mathbf{J}$, as the distribution precesses, the detection sensitivity changes, causing the image to undergo apparent rotation with increasing magnetic field strength. It should be stressed that though it may appear as if the velocity distribution of the fragments is rotating, this is not the case. The velocity distribution remains unchanged as a function of field strength, but the ionization probability in the probe step for products scattered at different angles changes as the $\mathbf{J}$ distribution rotates.

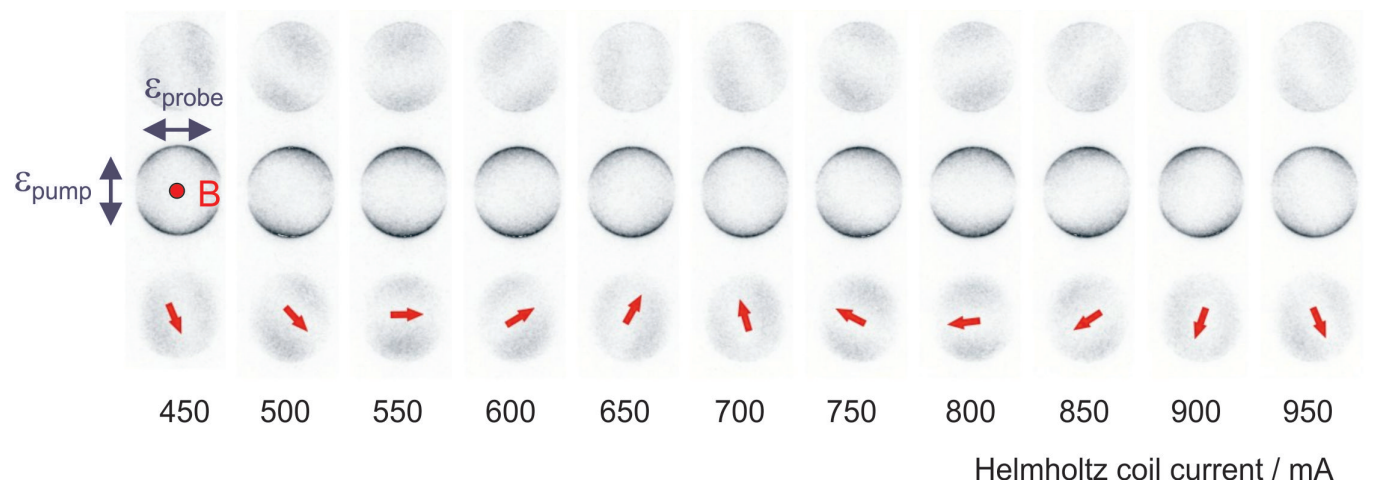

Figure 11: Sliced velocity-map images of $\mathrm{O}\left({ }^{1} \mathrm{D}\right)$ atoms formed in the $157 \mathrm{~nm}$ photolysis of $\mathrm{O}_{2}$, recorded as a function of magnetic field strength. As indicated in the first image, the magnetic field is applied perpendicular to the image plane, and both the pump and probe laser polarisations lie in the plane. Larmor precession of the strongly aligned $\mathrm{O}\left({ }^{1} \mathrm{D}\right)$ angular momentum distribution is visible in the images due to the alignment dependence of the REMPI detection step.

Using the experimental setup described above, the $\mathrm{O}\left({ }^{1} D\right)$ angular momentum distribution, and therefore its electron density distribution, may be made to rotate into any desired orientation in the lab frame by applying the appropriate magnetic field for the appropriate length of time. This opens up the intriguing possibility of using the technique to study chemical reactions involving $\mathrm{O}\left({ }^{1} D\right)$ as a function of atomic orbital orientation $[77,78,79]$. 


\section{Conclusions}

As we have seen, the preparation and characterisation of aligned and oriented atoms and molecules is now well established. The ability to overcome the random nature of the gas phase and to prepare samples of aligned and/or oriented atomic and molecular species has led to a broad range of innovative experiments in molecular and reaction dynamics. In the future, as the methodology for preparing aligned and oriented molecules moves out of the specialised research labs in which it was developed and into the mainstream, such approaches will become important in a variety of fields, ranging from gas-phase structure determination [76] to high harmonic generation [80], molecular spectroscopy $[81,82]$ and orbital tomography $[83,84,85]$.

\section{Acknowledgements}

The author thanks Katharine Reid, Henrik Stapelfeldt and Jochen Küpper for helpful discussions, and acknowledges funding via an ERC Starting Independent Researcher grant ImageMS, the EU ITN Network ICONIC, and the EPSRC Programme Grant EP/G00224X/1. 


\section{References}

[1] G. Scoles, Atomic and Molecular Beam Methods, (Oxford University Press, 1988).

[2] A. J. Orr-Ewing and R. N. Zare, 1994, Annu. Rev. Phys. Chem., 45, 315.

[3] R. N. Dixon, 1986, J. Chem. Phys., 85,1866.

[4] T. P. Rakitzis and R. N. Zare, 1999, J. Chem. Phys., 110(7), 3341.

[5] A. S. Bracker, E. R. Wouters, A. G. Suits and O. S. Vasyutinski, 1999, J. Chem. Phys., 110(14), 6749 .

[6] L. D. A. Siebbeles, M. Glass-Maujean, O. S. Vasyutinski, J. A. Beswick, and O. Roncero, 1994, J. Chem. Phys., 100, 3610.

[7] M. P. de Miranda and F. J. Aoiz, 2004, Phys. Rev. Lett., 93, 083201.

[8] M. P. de Miranda, F. J. Aoiz, V. Sáez-Rábanos, and M. Brouard, 2004, J. Chem. Phys., 121, 9830.

[9] N. E. Shafer-Ray, A. J. Orr-Ewing, and R. N. Zare, 1995, J. Phys. Chem., 99, 7591.

[10] M. J. Bass, M. Brouard, A. P. Clark, C. Vallance and B. Martinez-Haya, 2003, Phys. Chem. Chem. Phys., 5, 856.

[11] D. P. Pullmann, B. Friedrich, and D. R. Herschbach, 1990, J. Chem. Phys., 93, 3224.

[12] V.Aquilanti, D. Ascenzi, D. Cappelletti, and F. Pirani, 1994, Nature, 371, 399.

[13] V. Aquilanti, D. Ascenzi, M. de Castro Vitores, F. Pirani, and D. Cappelletti, 1999, J. Chem. Phys., 111, 2620.

[14] F. Pirani, D. Cappelletti, M. Bartolomei, V. Aquilanti, M. Scotoni, M. Vescovi, D. Ascenzi, and D. Bassi, 2001, Phys. Rev. Lett., 86, 5035.

[15] M. J. Weida and D. J. Nesbitt, 1994, J. Chem. Phys., 100, 6372.

[16] A. J. Orr-Ewing, 1996, J. Chem. Soc. Faraday Trans., 92, 881. 
[17] H. J. Loesch and A. Remscheid, 1990, J. Chem. Phys., 93, 4779.

[18] H. J. Loesch and J. Möller, 1992, J. Chem. Phys., 97, 9016.

[19] H. J. Loesch and A. Remscheid, 1993, J. Phys. Chem., 97, 2158.

[20] H. Li, K. J. Franks, R. J. Hanson, and W. Kong, 1998, J. Phys. Chem. A, 102(42), 8084.

[21] J. E. Mosch, S. A. Safron and J. P. Toennies, 1975, Chem. Phys., 8, 304.

[22] D.-C. Che, F. Palazzetti, Y. Okuno, V. Aquilanti, and T. Kasai, 2010, J. Phys. Chem. A, 114(9), 3280.

[23] P. W. Harland, W.-P. Hu, C. Vallance and P. R. Brooks, 1999, Phys. Rev. A, 60(4), 3138.

[24] J. Bulthuis, J. J. van Leuken, and S. Stolte, J. Chem. Soc. Faraday Trans., 1995, 91(2), 205.

[25] P. R. Brooks and E. M. Jones, 1966, J. Chem. Phys., 45(9), 3449.

[26] D. A. Blunt and P. W. Harland, 1994, J. Chem. Phys., 101(12), 11074.

[27] C. G. Aitken, D. A. Blunt, and P. W. Harland, 1995, Int. J. Mass Spectrom. Ion Proc., 149/150, 279.

[28] D. Normand, L. A. Lompré, and C. Cornaggia, 1992, J. Phys. B, 25, L497.

[29] B. Friedrich and D. Herschbach, 1995, Phys. Rev. Lett., 74(23), 4623.

[30] L. Holmegaard, J. H. Nielsen, I. Nevo, H. Stapelfeldt, F. Filsinger, J. Küpper, and G. Meijer, 2009, Phys. Rev. Lett., 102, 023001.

[31] F. Filsinger, J. Küpper, G. Meijer, L. Holmegaard, J. H. Nielsen, I. Nevo, J. L. Hansen, and H. Stapelfeldt, 2009, J. Chem. Phys., 131, 064309.

[32] I. Nevo, L. Holmegaard, J. H. Nielsen, J. L. Hansen, H. Stapelfeldt, F. Filsinger, G. Meijer, and J. Küpper, 2009, Phys. Chem. Chem. Phys., 11, 9912.

[33] H. Stapelfeldt and T. Seideman, 2003, Rev. Mod. Phys., 75, 543.

[34] T. Seideman, 1999, Phys. Rev. Lett., 83, 4971. 
[35] F. J. Aoiz, M. Brouard, P. A. Enriquez, and R. Sayos, 1993, J. Chem. Soc. Faraday Trans., 89, 1427.

[36] N. E. Shafer, A. J. Orr-Ewing, W. R. Simpson, H. Xu, and R. N. Zare, 1993, Chem. Phys. Lett., 212, 155; W. R. Simpson, A. J. Orr-Ewing, and R. N. Zare, ibid, 163.

[37] A. P. Clark, M. Brouard, F. Quadrini, and C. Vallance, 2006, Phys. Chem. Chem. Phys. 8, 5591.

[38] M. Brouard, R. Cireasa, A. P. Clark, F. Quadrini, and C. Vallance, 2007, Atomic polarisation in the photodissociation of polyatomic molecules, p 267-332 in Gas phase molecular reaction and photodissociation (edited by P. Kleiber and K. C. Lin, Research Signpost, 2007, ISBN 978-81-7895-305-2).

[39] S-M. Wu, D. Chestakov, G. Wu, X. Yang, C. Vallance, G. C. Groenenboom, W. J. van der Zande, and D. H. Parker, 2010, Mol. Phys., 108(7), 1145.

[40] P. R. Brooks, E. M. Jones, and K. Smith, 1969, J. Chem. Phys., 51(7), 3073.

[41] S. Kaesdorf, G. Schönhense, and U. Heinzmann, 1985, Phys. Rev. Lett., 54(9), 885.

[42] S. R. Ghandi and R. B. Bernstein, 1988, Z. Phys. D, 10, 179.

[43] S. R. Ghandi and R. B. Bernstein, 1990, J. Chem. Phys., 93, 4024.

[44] S. M. Wu, D. C. Radenovic, W. J. van der Zande, G. C. Groenenboom, C. Vallance, and R. N. Zare, 2011, Nature Chemistry, 3, 28.

[45] H. M. Lambert, A. A. Dixit, E. W. Davis, and P. L. Houston, 2004, J. Chem. Phys., 121, 10437.

[46] D. H. Parker, 2000, Acc. Chem. Res., 33(8), 563.

[47] A. T. J. B. Eppink, D. H. Parker, M. H. M. Jansenn, B. Buijsse, and W. J. van der Zande, 1998, J. Chem. Phys., 108(4), 1305.

[48] M. H. M. Janssen, J. W. G. Mastenbroek, and S. Stolte, 1997, J. Phys. Chem. A, bf 101, 7605. 
[49] J. J. Larsen, J. Sakai, C. P. Safvan, I. Wendt-Larsen, and H. Stapelfeldt, 1999, J. Chem. Phys., 111, 7774 .

[50] P. L. Houston, 1987, J. Phys. Chem., 91, 5388.

[51] M Brouard, P. O'Keeffe and C. Vallance, 2002, J. Phys. Chem. A., 106, 3629.

[52] A. J. Alexander and R. N. Zare, 1998, J. Chem. Ed., 75(9), 1105.

[53] V. Aquilanti, F. Pirani, D. Cappelletti, F. Vecchiocattivi, and T. Kasai, 2005, Theory of Chemical Reaction Dynamics, Nato Science Series II: Mathematics, Physics and Chemistry, $145,243$.

[54] C. T. Rettner and R. N. Zare, 1981, J. Chem. Phys., 75(7), 3636.

[55] L. Vattuone, L. Savio, F. Pirani, D. Cappelletti, M. Okada, and M. Rocca ,2010, Prog. Surf. Sci., 85, 92 .

[56] S. T. Ceyer, D. J. Gladstone, M. McGonigal, and M. T. Schulberg, in Investigations of Surfaces and Interfaces: Part A (Wiley, New York, 1993).

[57] L. B. F. Juurlink, P. R. McCabe, R. R. Smith, C. L. DiCologero, and A. L. Utz, 1999, Phys. Rev. Lett., 88, 868.

[58] J. Higgins, A. Conjusteau, G. Scoles, and S. L. Bernasek, 2001, J. Chem. Phys., 114, 5277.

[59] M. P. Schmid, P. Maroni, R. D. Beck, and T. R. Rizzo, 2003, Rev. Sci. Instrum., 74, 4110.

[60] R. D. Beck, P. Maroni, D. C. Papageorgopoulos, T. T. Dang, M. P. Schmid, and T. R. Rizzo, 2003, Science, 302, 98.

[61] D. R. Killelea, V. L. Campbell, N. S. Shuman, and A. L. Utz, 2008, Science, 319, 790.

[62] B. L. Yoder, R. Bisson, and R. D. Beck, 2010, Science, 329, 553.

[63] C. Vallance, R. G. A. R. Maclagan, and P. W. Harland, 1997, J. Phys. Chem. A, 101, 3505.

[64] P. W. Harland and C. Vallance, 'Positive ion electron impact ionization cross sections: theory and experiment', in Advances in Gas Phase Ion Chemistry, Volume 3, Ed. Nigel G. Adams and Lucia M. Babcock (J.A.I. Press Inc., 1998). 
[65] O. Gessner, Y. Hikosaka, B. Zimmerman, A. Hempelmann, R. R. Lucchese, J. H. D. Eland, P.-M. Guyon, and U. Becker, 2002, Phys. Rev. Lett., 98, 193002.

[66] Z. M. Wang, and D. S. Elliot, 2001, Phys. Rev. Lett., 87, 173001.

[67] Z. M. Wang and D. S. Elliott, Phys. Rev. Lett., 2000, 84, 3795.

[68] K. L. Reid, D. H. Leahy, and R. N. Zare, 1992, Phys. Rev. Lett., 68, 3527.

[69] H. Park, I. Konen, and R. N. Zare, 2000, Phys. Rev. Lett., 84, 3819.

[70] N. Bowering, T. Lischke, B. Schmidtke, N. Muller, T. Khalil, and U. Heinzmann, 2001, Phys. Rev. Lett., 86, 1187.

[71] M. Tsubouchi, B. J. Whitaker, L. Wang, H. Kohguchi, and T. Suzuki, 2001, Phys. Rev. Lett., 86,4500 .

[72] Y. Y. Yin, C. Chen, D. S. Elliott, and A. V. Smith, 1992, Phys. Rev. Lett., 69, 2353.

[73] K. L. Reid, 2003, Annu. Rev. Phys. Chem., 54, 397.

[74] L. Holmegaard, J. L. Hansen, L. Kalhøj, S. L. Kragh, H. Stapelfeldt, F. Filsinger, J. Küpper, G. Meijer, D. Dimitrovski, M. Abu-samha, C. P. J. Martiny and L. Bojer Madsen, 2010, Nature Phys., 6, 428.

[75] Y. Huismans, A. Rouzée, A. Gijsbertsen, J. H. Jungmann, A. S. Smolkowska, P. S. W. M. Logman, F. Lépine, C. Cauchy, S. Zamith, T. Marchenko, J. M. Bakker, G. Berden, B. Redlich, A. F. G. van der Meer, H. G. Muller, W. Vermin, K. J. Schafer, M. Spanner, M. Yu. Ivanov, O. Smirnova, D. Bauer, S. V. Popruzhenko, and M. J. J. Vrakking, 2011, Science, 331,61 .

[76] F. Krasniqi, B. Najjari, L. Stüder, D. Rolles, A. Voitkiv, and J. Ullrich, 2010, Phys. Rev. A, 81, 033411.

[77] C. T. Rettner and R. N. Zare, 1982, J. Chem. Phys., 77, 2416.

[78] M. H. M. Janssen, D. H. Parker, and S. Stolte, 1996, J. Phys. Chem., 100, 16066.

[79] R. N. Zare, 1998, Science, 279, 1875. 
[80] N. Hay, R. Velotta, M. Lein, R. de Nalda, E. Heesel, M. Castillejo, and J. P. Marangos, 2002, Phys. Rev. A, 65, 053805.

[81] K. J. Castle, J. Abbott, X. Peng, and W. Kong, 2000, J. Chem. Phys., 113(4), 1415.

[82] D. Arvanitis, N. Haack, G. Ceballos, H. Wende, K. Babershke, A. L. Ankudinov, and J. J. Rehr, 2000, J. Elecron Spectrosc. Relat. Phenom., 113, 57.

[83] S. Patchkovskii, Z. Zhao, T. Brabec, and D. M. Villeneuve, 2006, Phys. Rev. Lett., 97, 123003.

[84] S. Patchkovskii, Z. Zhao, T. Brabec, and D. M. Villeneuve, 2007, J. Chem. Phys., 126, 114306.

[85] E. V. van der Zwan, C. C. Chirila, and M. Lein, 2008, Phys. Rev. A, 78, 033410. 


\section{Figure Captions}

- Figure 1: (a) orientation of bond axes; (b) orientation of rotational angular momentum; (c) classical and quantum mechanical descriptions of alignment and orientation.

- Figure 2: Velocity distribution (open circles) and steric factor (closed circles) for $\mathrm{O}_{2}$ seeded $2.5 \%$ in He. The steric factor, $P$, is defined as $P=\left(w_{\perp}-w_{\|}\right) /\left(w_{\perp}+w_{\|}\right)$, where $w_{\perp}$ and $w_{\|}$are the weights for $\mathrm{O}_{2}$ molecules with their rotational angular momentum aligned perpendicular to and parallel to the beam axis, respectively. Adapted from reference [12]

- Figure 3: (a) Inhomogeneous electric field generated by a hexapole. The inscribed radius $\mathrm{r}_{0}$ is shown. (b) Principle of hexapole state selection and orientation: the hexapole selects upper Stark states from the initially randomly oriented molecular beam. These then pass adiabatically into a region of uniform electric field in order to achieve orientation in the lab frame.

- Figure 4: Velocity-map images of ground-state $\mathrm{Cl}$ atoms formed in the $308 \mathrm{~nm}$ photolysis of $\mathrm{Cl}_{2}$. Dissociation occurs via a pure perpendicular transition, and the images clearly show the resulting $\sin ^{2} \theta$ velocity distribution of the $\mathrm{Cl}$ atoms, with signal intensity peaking at right angles to the polarisation vector of the photolysis light (shown in white in the centre of the images). The atoms are detected by $(2+1)$ REMPI via the $4 p\left({ }^{4} P_{3 / 2}\right) \leftarrow{ }^{2} P_{3 / 2}$ transition.

- Figure 5: Laser alignment of iodobenzene molecules, detected through velocity-map imaging of I atoms produced when the molecule is Coulomb exploded by a high intensity femtosecond probe pulse. In (a) the alignment laser is turned off, and the distribution of I atoms is isotropic. In (b)-(d) the alignment laser is turned on, and the iodobenzene is strongly aligned, more so for low rotational states (image $(\mathrm{c})$ ) than high rotational states (image $(\mathrm{d})$ ). The alignment laser polarisation $\epsilon_{\text {align }}$ is indicated to the right of the images. Adapted from reference [30].

- Figure 6: Velocity-map images recorded for I* fragments following $266 \mathrm{~nm}$ dissociation of hexapole state-selected and oriented $\mathrm{CD}_{3} \mathrm{I}$ in its $|J K M\rangle=|111\rangle$ state. The direction of the orienting field and photolysis laser polarisation are shown to the left of the images, and the images recorded from left to right illustrate the effect of increasing the orienting field over the range from 0 to $1600 \mathrm{~V} \mathrm{~cm}^{-1}$. Adapted from reference [48]. 
- Figure 7: (a)Proposed mechanisms for glyoxal dissociation leading to $\mathbf{J} \| \mathbf{v}$ (left) and $\mathbf{J} \perp \mathbf{v}$ (right); (b) Doppler profiles for CO fragments formed in $(v=0, J=51)$. As explained in the text, the characteristic peak shapes observed for the $\mathrm{P}, \mathrm{Q}$, and $\mathrm{R}$ transitions allow the two mechanisms to be distinguished. Adapted from reference [50].

- Figure 8: Velocity-map images of $\mathrm{O}\left({ }^{1} D\right)$ from the photodissociation of $\mathrm{O}_{2}$ at $157 \mathrm{~nm}$. As described in the text, images were acquired on two REMPI transitions using two experimental geometries defined by the pump and probe laser propagation and polarisation axes.

- Figure 9: Measurement of the minimum field required to achieve orientation following hexapole state selection of symmetric top molecules. Molecules state-selected in a first hexapole will only focus in a second hexapole if the field strength in the intervening uniform $\mathrm{C}$ field is sufficient to maintain the distribution of Stark states.

- Figure 10: Photoelectron distributions recorded from benzonitrile molecules that are: (a) randomly oriented; (b) oriented in one dimension; and (c) oriented in three dimensions. In the case of three dimensional orientation the nodal plane of the molecular orbital in the plane of the molecule is revealed. Adapted from reference [74].

- Figure 11: Sliced velocity-map images of $\mathrm{O}\left({ }^{1} \mathrm{D}\right)$ atoms formed in the $157 \mathrm{~nm}$ photolysis of $\mathrm{O}_{2}$, recorded as a function of magnetic field strength. As indicated in the first image, the magnetic field is applied perpendicular to the image plane, and both the pump and probe laser polarisations lie in the plane. Larmor precession of the strongly aligned $\mathrm{O}\left({ }^{1} \mathrm{D}\right)$ angular momentum distribution is visible in the images due to the alignment dependence of the REMPI detection step. 